\title{
Dynamic Surface Tension and Diffusivity Measurements of NG-CSSX Next Generation Solvent
}

\section{F. F. Fondeur}

T. B. Peters

May 2014

SRNL-STI-2013-00733, Revision 0 
SRNL-STI-2013-00733

Revision 0

\section{DISCLAIMER}

This work was prepared under an agreement with and funded by the U.S. Government. Neither the U.S. Government or its employees, nor any of its contractors, subcontractors or their employees, makes any express or implied:

1. warranty or assumes any legal liability for the accuracy, completeness, or for the use or results of such use of any information, product, or process disclosed; or

2. representation that such use or results of such use would not infringe privately owned rights; or

3. endorsement or recommendation of any specifically identified commercial product, process, or service.

Any views and opinions of authors expressed in this work do not necessarily state or reflect those of the United States Government, or its contractors, or subcontractors.

\section{Printed in the United States of America \\ Prepared for U.S. Department of Energy}




\section{Dynamic Surface Tension and Diffusivity Measurements of NG-CSSX Next Generation Solvent}

F. F. Fondeur

T. B. Peters

May 2014 


\section{REVIEWS AND APPROVALS}

\section{AUTHORS:}

F. F. Fondeur, Separation \& Actinide Science

Date

T. B. Peters, Advance Characterization \& Process

Date

TECHNICAL REVIEW:

T. E. White, Spectroscopy, Separation \& Log Reviewed per E7 2.60

Date

S. D. Fink, E\&CPT Research Programs

Date

APPROVAL:

F.M. Pennebaker, Manager

Date

Advanced Characterization \& Processing

S.L. Marra, Manager

Date

E\&CPT Research Programs 
SRNL-STI-2013-00733

Revision 0

\section{EXECUTIVE SUMMARY}

Since 2008, the Savannah River Site has processed more than four million gallons of radioactive supernatant. To continue compliance with downstream processes, MCU is switching to the improved solvent called Next Generation Solvent (NG-CSSX). The new solvent formulation promises higher cesium removal efficiency and laboratory testing suggests the potential of higher processing rates. Similarly, laboratory testing demonstrated improvements in separation of the solvent from the effluent aqueous streams. To complete implementation at MCU, additional work was needed to develop methods that can measure physical properties relevant to the performance of the new solvent. This work measures two relevant physical properties: static interfacial tension and diffusvity. Both properties play a relevant role in mixing and separating immiscible liquids. This work evaluated the impact of changes in composition or aqueous phase on these physical properties to gauge the sensitivity of the system to the losses of chemical components.

This work found that the suppressor, TiDG, when it contacts $10 \mathrm{mM}$ boric acid ionizes and lowers the interfacial tension between NGS-CSSX and boric acid. The interfacial tension lowering is not sufficient to stop NGS-CSSX droplet coalescing. It was also found that the modifier enhances carry-over in the salt simulant of the NGS-CSSX solvent probably through the alcohol group in the modifier molecule. The extractant also assisted in the salt simulant carry-over of the NGS-CSSX after contacting salt simulant.

Several literature-published adsorption models were evaluated for fitting the measured static interfacial tension data. It was found that both empirical and thermodynamically derived models fit the data. Because of the limited dataset, a true discrimination of the fitting models was not possible.

It was also found that increasing the Isopar ${ }^{\circledR} \mathrm{L}$ concentration in NGS increased the self-diffusivity of the NGS components in NGS-salt simulant emulsions. The diffusion NMR method was able to show and determine the amount of branched and cyclic molecules in Isopar ${ }^{\circledR} \mathrm{L}$. The method provided an estimate of the NGS viscosity in NGS-salt solution emulsions. The method also gave an estimate of the dynamic interfacial tension of NGS droplets in NGS-salt solution emulsions.

The data collected here seems to indicate that the relatively high levels of MaxCalix may lead to excess NGS-CSSX droplets carry over to the salt simulant. It was determined this effect is mainly due to the concentration level and not to additional specific chemical interactions between MaxCalix and the salt simulants. The effect is more pronounced at high energy densities of mixing. 


\section{TABLE OF CONTENTS}

LIST OF TABLES. vii

LIST OF FIGURES viii

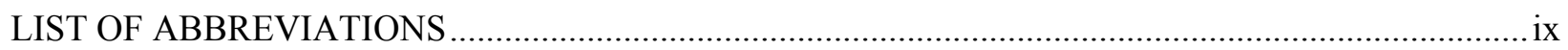

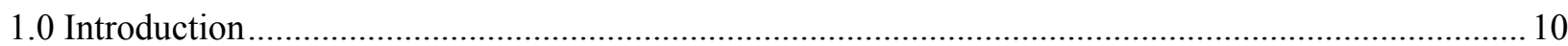

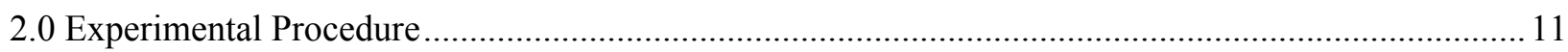

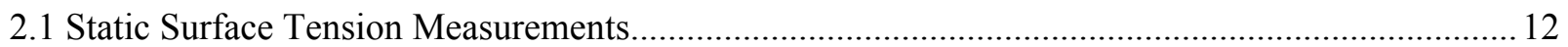

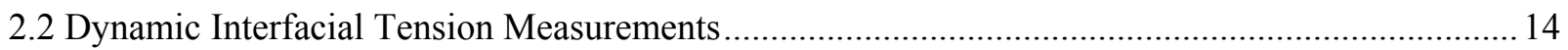

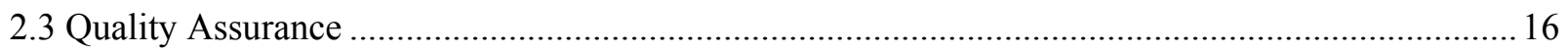

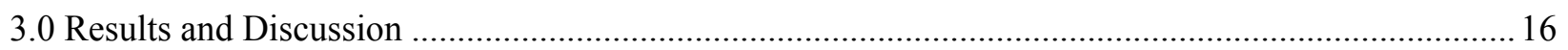

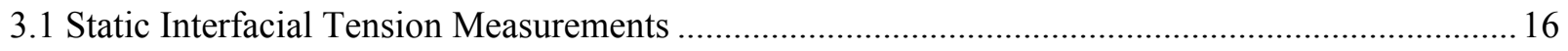

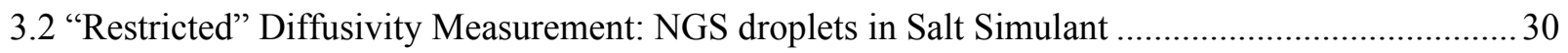

3.3 Diffusivity Measurement: NGS Droplets in $10 \mathrm{mM}$ Boric Acid................................................... 33

3.4 Diffusivity Measurement: Salt Simulant or Boric Acid Droplets in NGS Solvent ......................... 33

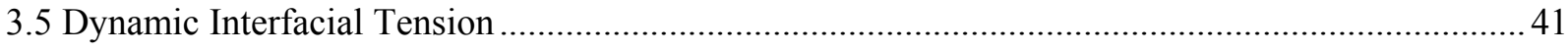

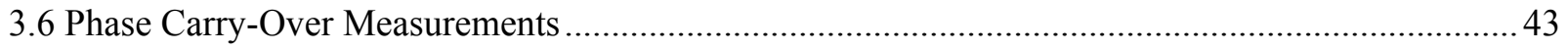

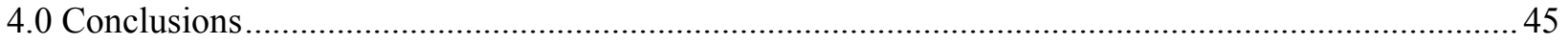

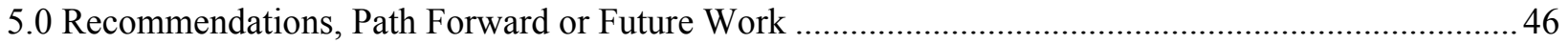

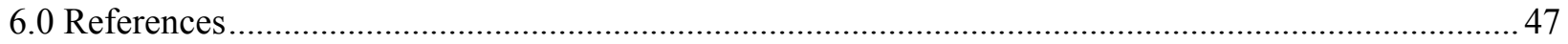

Appendix A . A Typical Exponential Decay fit to the NMR Data to extract the Diffusivity Constant...A-1 


\section{LIST OF TABLES}

Table 2-1. Solvent Formulations. 12

Table 2-2. The Diffusion Coefficient of Standard Samples ${ }^{11}$ .15

Table 3-1. Results from Interfacial Tension (IFT) Measurements. 18

Table 3-2. The Hansen's solubility parameters for the NG-CSSX components and corresponding SFT . 21

Table 3-3. The Hansen's solubility parameters and the predicted SFT estimation of the NGS samples...21

Table 3-4. Parameters values from Eq. 3 that best fit the data in Table 3-1 ........................................... 22

Table 3-5. Fitting results of the NG-CSSX/Salt Simulant with Wilson's model................................... 24

Table3-3-6 Fitting results of the NG-CSSX/Boric Acid with Wilson's Model........................................24

Table 3-7. Prediction performance of the Hildebrand-Scott model for NG-CSSX/Salt simulant (all units in dyn $/ \mathrm{cm}) . \mathrm{A}_{2}=27.5$ and $\mathrm{A}_{1}=32$.

Table 3-8. Prediction performance of the Hildebrand-Scott model for NG-CSSX/Boric Acid (all units in dyn $/ \mathrm{cm}) . A_{2}=40$ and $A_{1}=0$. 25

Table 3-9. Mole fraction solubility of NGS in salt simulant and vice versa as predicted in Eq. 6 ...........26

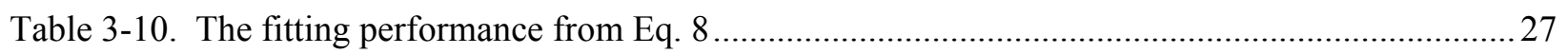

Table 3-11. Estimates of the IFT values of the "Balance of Solvent"and Isopar ${ }^{\mathbb{R}}$ L in Salt Solution and in

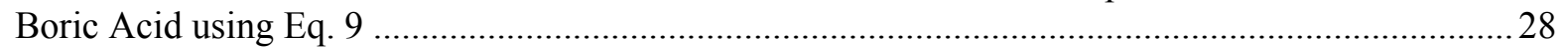

Table 3-12. The estimated solubility of NGS in salt simulant based on Eq. 10 ....................................29

Table 3-13. Prediction of the reduced IFT values (Table 3-1) using Eq. 11 and Eq. 12 assuming the modifier is the surface active agent in contact with salt simulant. .................................................... 30

Table 3-14. NGS diffusivity $\left(\mathrm{m}^{2} / \mathrm{s}\right)$ in Salt Simulant and Boric acid diffusivities .................................. 32

Table 3-15 Relevant parameters determined from the fit in Fig. 3.3 ….................................................. 32

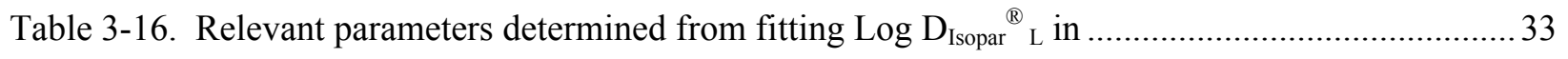

Table 3-17. Salt simulant and boric acid diffusivity $\left(\mathrm{m}^{2} / \mathrm{s}\right)$ in NGS solvent after dispersion ................... 34

Table 3-18. Relevant parameters determined from fitting $\log \mathrm{D}_{\text {salt simulant }}$ in ........................................ 34

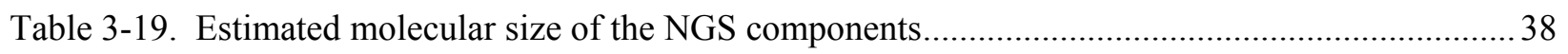

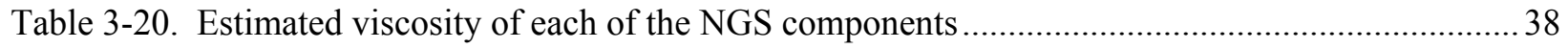

Table 3-21. The solvent (CSSX) viscosity in Pa*s of samples from Ref. 38 (1000 cP = Pa*s). Also shown the predicted modifier-extractant-suppressor combo viscosity ..............................................39

Table 3-22. Estimation of the solvent viscosity with the modified Einstein equation proposed by Guth ${ }^{39}$. All viscosity data shown in $\mathrm{cP}\left(\mathrm{mPa}^{*} \mathrm{~s}\right)$..... 
Table 3-23. The Calculated Dynamic Interfacial Tension between Salt Simulant ................................. 42

Table 3-24. Percent Isopar ${ }^{\circledR} \mathrm{L}$ in boric acid and in salt simulant after mixing ...................................... 43

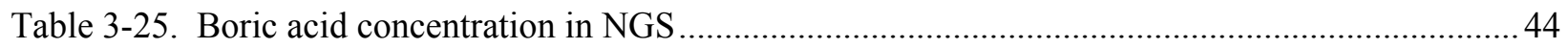

Table 3-26. The Effect of the NGS Components on NGS Solubility in Salt Simulant ........................... 44

\section{LIST OF FIGURES}

Figure 2-1. DI Water Calibration of the Surface Tensiomat model \# 21(average measure temperature $\left.21.5^{\circ} \mathrm{C}\right)$

Figure 2-2. A plot of diffusivity distribution (y-axis) versus the H-NMR spectrum of the emulsion (xaxis).

Figure 3-1. Influence of Modifier and MaxCalix on the interfacial tension between NGS and Salt Simulant

Figure 3-2. Influence of TiDG on the static interfacial tension between NGS and Boric acid. .19

Figure 3-3. Fitting results of the Log $\mathrm{D}_{\text {Isopar }}^{\circledR} \mathrm{L}$ of NGS droplets in Salt Simulant ..................................... 32

Figure 3-4. H-NMR spectrum of the component in Isopar ${ }^{\circledR} \mathrm{L}$ that diffuses at $3.89 \mathrm{E}-10 \mathrm{~m}^{2} / \mathrm{s} \ldots \ldots \ldots \ldots . . . .36$

Figure 3-5. H-NMR spectrum of the component in Isopar $^{\circledR} \mathrm{L}$ that diffuses at $3.47 \mathrm{E}-10 \mathrm{~m}^{2} / \mathrm{s} \ldots \ldots \ldots \ldots \ldots . . . . .36$

Figure 3-6. H-NMR spectrum of the component in $\operatorname{Isopar}^{\circledR} \mathrm{L}$ that diffuses at $2.29 \mathrm{E}-10 \mathrm{~m}^{2} / \mathrm{s} \ldots \ldots \ldots \ldots \ldots . . . . . .37$

Figure 3-7. Histogram (or concentration) of diffusing species in salt solution/NGS emulsions ...............37 


\section{LIST OF ABBREVIATIONS}

$\begin{array}{cl}\text { CSSX } & \text { Caustic-Side Solvent Extraction } \\ \text { DI } & \text { Deionized } \\ \text { DWPF } & \text { Defense Waste Processing Facility } \\ \text { IFT } & \text { Interfacial Tension } \\ \text { MCU } & \text { Modular Caustic-Side Solvent Extraction Unit } \\ \text { NGS } & \text { Next Generation Solvent } \\ \text { NMR } & \text { Nuclear Magnetic Resonance } \\ \text { RSD } & \text { relative standard deviation } \\ \text { SE } & \text { Strip Effluent } \\ \text { SFT } & \text { Surface Tension } \\ \text { SRNL } & \text { Savannah River National Laboratory } \\ \text { SRS } & \text { Savannah River Site } \\ \text { TTQAP } & \text { Task Technical and Quality Assurance Plan } \\ \text { TTR } & \text { Task Technical Request }\end{array}$




\subsection{Introduction}

Since April 2008, the Savannah River Site (SRS) has processed more than four million gallons of supernatant at the Modular Caustic-Side Solvent Extraction Unit (MCU). At MCU, a train of 18 centrifugal contactors ( 7 for extraction, 2 for scrubbing, 7 for stripping, and 2 for solvent washing) are connected in series and provide both mixing and separation of two immiscible streams: a multi-component organic solvent and an aqueous salt supernatant.

The Next Generation Solvent (NGS) will be used to process dissolved salt cake after September 2013. While decontamination operations with the current solvent (using BOBCalixC6 as the extractant $)^{i}$ have been underway since 2008, the chemical changes from the current solvent to the new solvent formulation (NGS-MCU) require testing to mitigate risk and progress the Technical Maturation Level. The NGS contains MaxCalix ${ }^{\text {ii }}$ and $\mathrm{TiDG}^{\mathrm{iii}}$ as the extractant and suppressor respectively.

The existing Caustic-Side Solvent Extraction (CSSX) solvent (heel layer) was combined with a specially prepared compound of four components, generating a "Blend" solvent containing both the old solvent components, as well as new ones. ${ }^{\text {iv }}$ Usage of the Blend solvent should reduce the curies of ${ }^{137} \mathrm{Cs}$ sent to Saltstone, increase the waste operating window, and still retain the old (CSSX) solvent's low organic carryover to DWPF and Saltstone. A recent laboratory testing with the $2 \mathrm{~cm}$ contactors ${ }^{1,2}$ demonstrated less than $3.3 \mathrm{mg} / \mathrm{L}$ Isopar ${ }^{\circledR} \mathrm{L}$ in the SE stream and less than $140 \mathrm{mg} / \mathrm{L}$ in the DSS stream carryover with this Blend solvent.

Non-radioactive contact testing of NGS and the Blend solvent with aqueous solutions revealed excess secondary phase (aqueous) carry over in the organic liquid at high solvent flow rates. ${ }^{2}$ The aqueous carry-over was more noticeable when contacts were made with caustic salt simulant. Personnel who conducted those tests suspect a favorable interaction between water molecules and the new components in NGS (such as MaxCalix and $\mathrm{DCiTG}^{\mathrm{v}}$ ) that could slow down the dewatering rate of NGS (or the aggregation rate of water). The NGS-MCU formulation replaced the DCiTG suppressor with TiDG to reduce carryover of this species to the aqueous streams.

There are various mechanisms that may explain the excess water retention of NGS, or NGS-MCU, including at the macroscopic scale large capillary number (i.e., lower interfacial tension), larger than expected interfacial viscosity (despite the higher Isopar ${ }^{\circledR} \mathrm{L}$ content of these solvent formulations), or interfacial elasticity that prevents the solvent draining from the coalescing of salt simulant droplets. Also at the microscopic level, strong interactions (such as hydrogen bonding) between water molecules or water

${ }^{\mathrm{i}}$ BOBCalixC6 stands for calix[4] arene-bis-(tert-octylbenzo)-crown-6.

ii MaxCalix stands for 1,3-alt-25,27-bis(3,7-dimethyloctyl-1-oxy) calix[4]arene-benzocrown-6.

iii TiDG stands for N,N',N"-tris (3,7-dimethyloctyl) guanidine.

iv The Blend solvent nominally consists of $0.5 \mathrm{M}$ modifier, 46.5 mM MAXCalix, 3.5 mM BOBCalixC6, 1.5 mM TOA, $3 \mathrm{mM}$ TiDG in Isopar $^{\circledR} \mathrm{L}$.

${ }^{v}$ DCiTG stands for 1,3-dicyclohexyl-2-(11-methyldodecyl)guanidine, alternatively referred to as N, N'-dicyclohexylN"-isotridecylguanidine. 
containing species with the guanidine or MaxCalix molecules in the solvents can enhance water retention.

Testing has been conducted to ensure the processability, mixing, and other operating requirements of the Blend solvent are appropriate and to better understand the physical characteristics of NGS. SRNL, Parsons, and other laboratories have conducted numerous analytical studies that determined and confirmed the hydraulics, compatibility, and mass transfer performance of the new solvent. ${ }^{3,4,5}$ This work evaluates other physical properties that are significant to the understanding of the performance of this solvent. This work outlines the efforts to determine the solvent's component concentration effects on the static and dynamic interfacial tension of the NGS solvent. This work was originally proposed in an early Task Technical and Quality Assurance Plan (TTQAP). ${ }^{6}$ While the TTQAP outlines an extensive body of work, budget and time limitations forced SRNL to reduce the scope to what is described in this document.

\subsection{Experimental Procedure}

Testing objectives include fitting the data to known IFT (interfacial tension) models for future prediction and to determine the chemical components that significantly affect the IFT of the NG-CSSX solvent.

To examine the effects of the individual solvent components that make up the NG-CSSX solvent on the interfacial tension, a statistically designed matrix of solvents was generated of 30 different formulations. From this set of 30, eight were chosen for measurement. These eight were prepared, and have their compositions listed in Table 2-1, as well as their measured densities. These eight compositions represent the corners of a three dimensional cube in the compositional space of the components that make up the solvent. The output, such as interfacial tension (IFT) is fitted to a lineal polynomial that includes interactions. For example, IFT $=\mathrm{aX}+\mathrm{bY}+\mathrm{c} \mathrm{XY}$ (with no quadratic terms considered). Recall this sample matrix is not optimized for discriminating different models. It is only sufficient for determining which chemical constituent in NGS impacts the IFT value.

A full factorial design would have required 16 experiments (or $2^{4}$ ) and one center point experiment for detecting any curvature in the data. Expecting that no significant interactions between three components on diffusivity, the number of tests in the testing protocol was reduced. Based on that assumption, testing protocol only considered eight experiments (or $2^{4-1}$ ) that provides a full factorial for the modifier, extractant, and suppressor effects. With this design, three-way interactions are assumed insignificant; the effect from any factor is purely due to that factor alone. Note that the concentrations of Isopar ${ }^{\mathbb{B}} \mathrm{L}$ and modifier are not independent variables but rather are constrained by the sum total of the solvent concentration constrained by the total liquid volume fixed to $120 \mathrm{~mL}$. More specifically, the Isopar ${ }^{\circledR} \mathrm{L}$ level is determined or bounded by $1=$ 
$\sum_{i=1}^{4} x_{i} \times d_{\text {solvent }} / d_{i}$ where $x_{i}$ stands for the mole fraction of $i^{\text {th }}$ component, $d_{\text {solvent }}$ and $d_{\mathrm{i}}$ stands for the solvent and it's pure components density respectively. The data can be analyzed as a mixture rather than an aggregate of components as assumed when using fractional factorial analysis. Thus, when the modifier level in the mixture is high, the corresponding level in the Isopar ${ }^{\circledR} \mathrm{L}$ is low. Because the data spans different levels of magnitude (1E-10 and 1E-11), the measured diffusivity data was transformed with the base-10 logarithm function before it was analyzed with the JMP ${ }^{\complement}$ Software version 8.0. With this transformation, the range of the data is reduced and minimizing (or leveraging) the high magnitude against the low magnitude data. Thereby, this reduces the likelihood of computer error when centering, normalizing, and computing the data.

Table 2-1. Solvent Formulations

\begin{tabular}{|c|c|c|c|c|}
\hline Solvent ID & Modifier (M) & Extractant (M) & Suppressor (M) & Density (g/mL) \\
\hline NGS-1 & 0.5 & 0 & 0 & $0.833 @ 22.5^{\circ} \mathrm{C}$ \\
\hline NGS-2 & 0.5 & 0 & 0.005 & $0.840 @ 22.5^{\circ} \mathrm{C}$ \\
\hline NGS-5 & 0.5 & 0.05 & 0.005 & $0.855 @ 22.0^{\circ} \mathrm{C}$ \\
\hline NGS-6 & 0.75 & 0.05 & 0 & $0.885 @ 22.0^{\circ} \mathrm{C}$ \\
\hline NGS-7 & 0.75 & 0 & 0 & $0.868 @ 22.0^{\circ} \mathrm{C}$ \\
\hline NGS-11 & 0.75 & 0.05 & 0.005 & $0.886 @ 22.5^{\circ} \mathrm{C}$ \\
\hline NGS-13 & 0.75 & 0 & 0.005 & $0.872 @ 22.5^{\circ} \mathrm{C}$ \\
\hline NGS-18 & 0.63 & 0.025 & 0.0025 & $0.865 @ 22.5^{\circ} \mathrm{C}$ \\
\hline
\end{tabular}

In each case, $120 \mathrm{~mL}$ of solvent was prepared, using gravimetrically measured amounts of modifier, extractant, and suppressor. Isopar ${ }^{\mathbb{B}} \mathrm{L}$ was then added in volumetric glassware such that the precise final volume $(120 \mathrm{~mL})$ of each solvent was prepared.

\subsection{Static Surface Tension Measurements}

The TTQAP states that a pendant drop method for interfacial tension would be used. However, this type of instrument was not available, and as a result, a du Nouy method was used instead.

Once all eight solvents were prepared, researchers performed surface tension measurements. Before any of the prepared solvents were used, the tension component of the instrument ${ }^{\mathrm{vi}}$ was calibrated using a known mass (so the total system is kept constant between measurements), followed by a quadruple single phase measurement of DI water (at $21.5^{\circ} \mathrm{C}$ ). The four measurements gave a measured average of $76.6(0.81 \% \mathrm{RSD})$ dynes $/ \mathrm{cm}$, which was temperature corrected to an average value of 71.8 dynes $/ \mathrm{cm}$. This value was compared to standard set of reference data ${ }^{7}$ which predicted an absolute surface tension of 72.6 dynes $/ \mathrm{cm}$. The difference between the measured and reference

\footnotetext{
${ }^{\text {vi }}$ Fisher Surface Tensiomat Model 21, which uses a du Nouy method. The analytical methodology described in the
} users manual was used. 
SRNL-STI-2013-00733

Revision 0

value $(\sim 1.1 \%)$ was considered to be an indication of acceptable performance and precision. See Figure 2-1.

Figure 2-1. DI Water Calibration of the Surface Tensiomat model \# 21(average measure temperature $21.5^{\circ} \mathrm{C}$ )

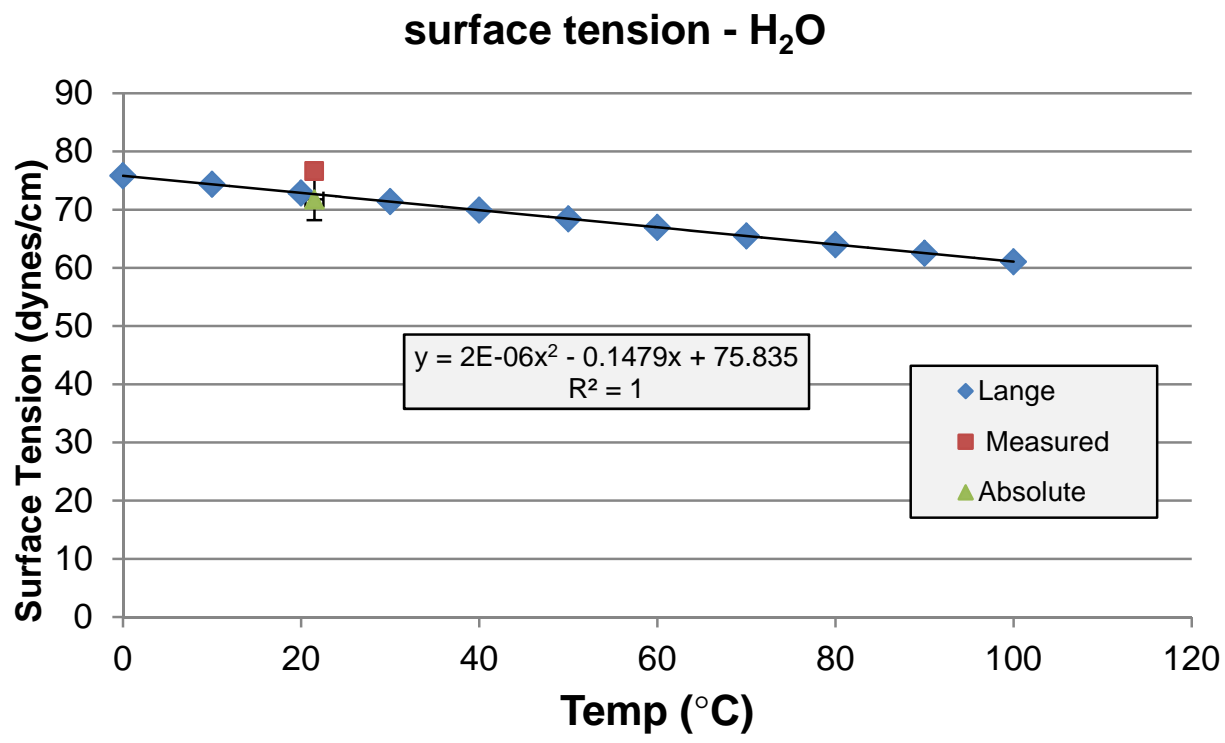

After the instrument was calibrated, all measurements were performed using the same interfacial tension method. The general method follows.

First, a clean $50 \mathrm{~mL}$ glass beaker was filled with $30 \mathrm{~mL}$ of the aqueous solution. Then, the du Nouy ring was placed and aligned such that the ring was $\sim 1 / 8$ " below the surface. The torsion arm is aligned with the zero mark, and the measurement dial is set to zero. $15 \mathrm{~mL}$ of the solvent phase was carefully layered on top of the aqueous layer, such that there was minimal disturbance or mixing of the two phases. The measurement dial was reset to zero to correct for the minor perturbation.

The torsion on the arm was then slowly adjusted upwards, while at the same time, lowering the beaker height and making sure the torsion arm was aligned with the zero mark. This requires two hands, and a close view on the zero mark. The measured interfacial surface tension corresponds to the point where the du Nouy ring bursts upwards entirely from the lower, denser phase, into the lighter top phase.

Each of the eight solvent preparations were measured against each of two phases; one designed to mimic the salt waste solution ( $\sim 5 \mathrm{M}$ sodium $\mathrm{V}-5 / 10$ salt simulant ${ }^{8}$ ), and the other the strip acid solution (0.01 M boric acid) used at MCU. In each case, duplicate 
measurements were performed. Between measurements, the $50 \mathrm{~mL}$ beakers were thoroughly cleaned with isopropanol, DI water, then isopropanol and then force-air dried in that order. The du Nouy rings were cleaned with isopropanol, DI water, then isopropanol, and then finally flame-dried.

\subsection{Dynamic Interfacial Tension Measurements}

After the static surface tension measurements, the samples from the eight solvent formulations were contacted with aqueous phase (salt simulant or $0.01 \mathrm{M}$ boric acid) at a four to one volume ratio (aqueous to solvent) in a $30 \mathrm{~mL}$ Teflon ${ }^{\circledR}$ bottle to simulate the liquid-liquid phase mixing in the centrifugal contactors in MCU. Then, the tip of a homogenizer ${ }^{\text {vii }}$ was inserted into the heterogeneous mixture. The tip's penetration depth was kept the same for every sample ( $3 \mathrm{~mm}$ off from the bottom of the bottle). The rotor spun at $15,000 \mathrm{rpm}$ for 1.5 minutes to generate an emulsion. In a previous test with CSSX solvent using these conditions, the obtained mean particle distribution $(15 \mu \mathrm{m})^{9}$ was similar to the mean particle size distribution observed in the scale-up test with the NG-CSSX solvent $(<18 \mu \mathrm{M}) .{ }^{10}$ After waiting for 20 seconds, two visibly distinctive emulsified (turbid) liquid layers formed with the cloudy organic layer sitting on top of the cloudy aqueous layer. At this point, approximately $1.5 \mathrm{~mL}$ of liquid was drawn from each layer for diffusion analysis. In the cases of the solvent contacting boric acid, the rotor spun at $500 \mathrm{rpm}$ for 20 seconds to lessen the foaming tendency.

Diffusivity measurements were conducted in the narrow bore of a 7 Tesla magnet equipped with a Bruker Avance $300 \mathrm{MHz}$ spectrometer that included a gradient board. While the actual task is more involved, a brief general method follows. Approximately $1 \mathrm{~mL}$ of emulsified solution was placed in a $5 \mathrm{~mm}$ ID cylindrical glass tube from Oxford Inc. certified for NMR analysis. The glass cylinder was then placed inside a set of metallic coils and the whole probe was then inserted inside the 7 Tesla magnet. The sample was then irradiated with radio waves $(\mathrm{kHz})$ and after waiting for a fixed time period to allow for self-diffusion to take place, the remaining magnetization was read. During the waiting period, a magnetic field gradient $(\sim 45 \mathrm{Gauss} / \mathrm{mm})$ was applied to the sample along the axial direction of the cylindrical tube. This magnetic field gradient codifies the spatial location and phase of the magnetism from each nucleus.

The NMR method was first calibrated with a standard containing $99 \% \mathrm{D}_{2} \mathrm{O}$ and $1 \% \mathrm{H}_{2} \mathrm{O}$ by volume. The standard sample measured $1.76 \mathrm{E}-9 \mathrm{~m}^{2} / \mathrm{s}$ at $20^{\circ} \mathrm{C}$. The literature data indicates the standard should measure $1.91 \mathrm{E}-9 \mathrm{~m}^{2} / \mathrm{s}$ at $25^{\circ} \mathrm{C}$ (see Table 2-2). ${ }^{11}$ Given that diffusivity is temperature sensitive, the difference of $8 \%$ is probably due to the lower temperature condition during our measurement. Five additional diffusivity measurements

\footnotetext{
vii The homogenizer, from Biospec Products, consists of a cylinder (rotor) inside another cylinder (stator). The rotor and stator inside diameters are $9 \mathrm{~mm}$ and $10.7 \mathrm{~mm}$ respectively. At 15,000 rpm, the shear rate is 79,412 per second.
} 
of the standard 14 hours later averaged to $1.773 \mathrm{E}-9 \mathrm{~m}^{2} / \mathrm{s}$ with a percent relative standard deviation of 2.62 .

Table 2-2. The Diffusion Coefficient of Standard Samples ${ }^{11}$

\begin{tabular}{|c|c|c|}
\hline Sample & Temperature $\left({ }^{\circ} \mathbf{C}\right)$ & D $\left(\mathbf{1 0}^{-\mathbf{9}} \mathbf{m}^{\mathbf{2}} / \mathbf{s}\right)$ \\
\hline $\mathrm{H}_{2} \mathrm{O}$ & 20.0 & 2.03 \\
\hline $\mathrm{H}_{2} \mathrm{O}$ & 25.0 & 2.3 \\
\hline $\mathrm{H}_{2} \mathrm{O}$ in $\mathrm{D}_{2} \mathrm{O}$ & 25.0 & 1.91 \\
\hline
\end{tabular}

For binary suspensions, a typical diffusivity $\left(\mathrm{m}^{2} / \mathrm{s}\right)$ versus H-NMR spectrum is shown Figure 2-2.

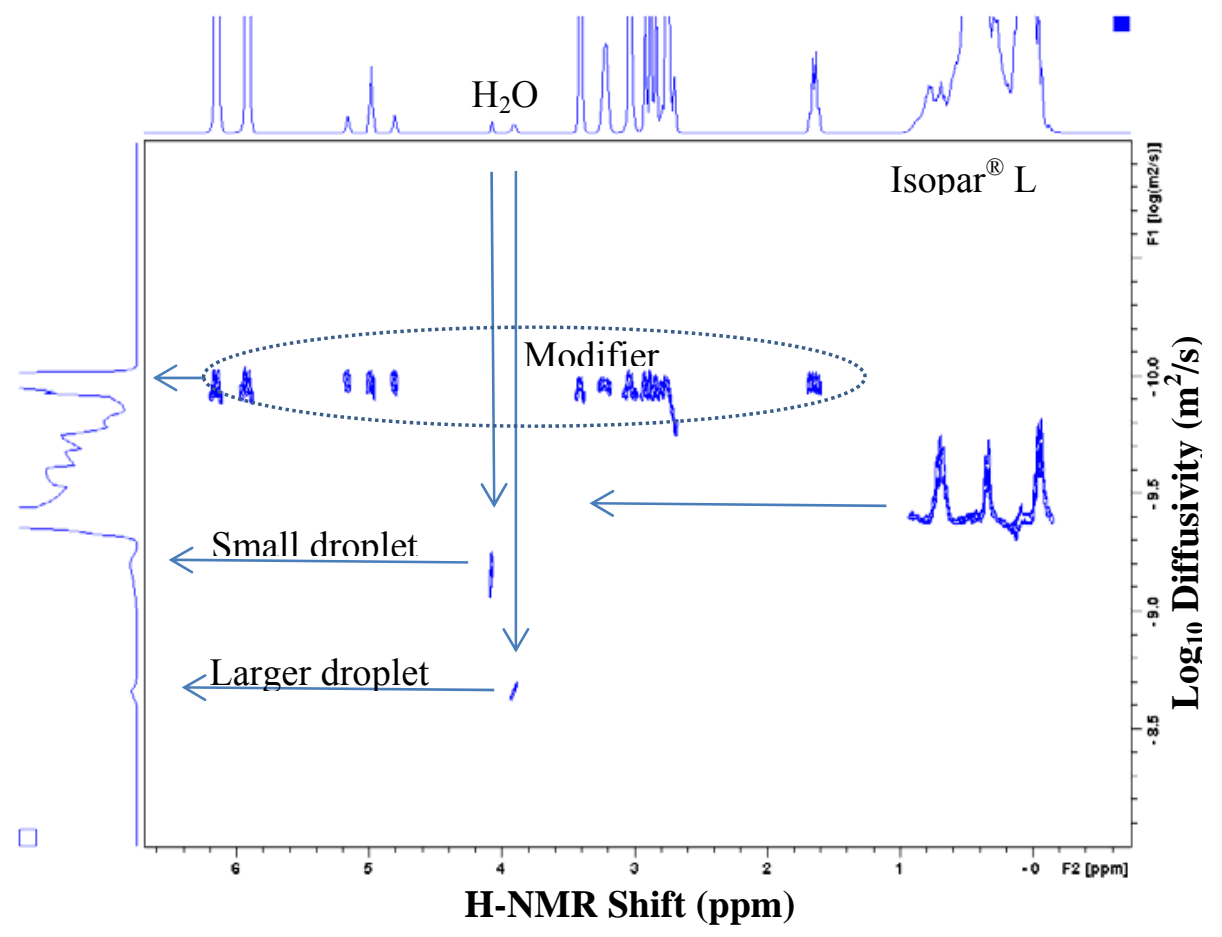

Figure 2-2. A plot of diffusivity distribution (y-axis) versus the H-NMR spectrum of the emulsion ( $x$-axis).

As shown in Figure 2-2, the diffusivity constant was measured for all the different components in the emulsion. For salt simulant or boric acid suspensions in NGS solvent, the diffusivity measurement reported is that for water associated with "small" droplets. For solvent suspensions in salt simulant or boric acid, the diffusivity constant reported is that of Isopar $^{\circledR} \mathrm{L}$. 
Phase carryover was estimated from the single H-NMR spectrum taken from each emulsion sample before conducting the diffusion experiments. The area under the water and Isopar ${ }^{\circledR} \mathrm{L}$ peaks was measured and the ratio taken to estimate the relative proportion of the entrained component in the aqueous or NGS phases.

\subsection{Quality Assurance}

Requirements for performing reviews of technical reports and the extent of review are established in manual E7 2.60. SRNL documents the extent and type of review using the SRNL Technical Report Design Checklist contained in WSRC-IM-2002-00011, Rev. 2.

\subsection{Results and Discussion}

\section{1 $\underline{\text { Static Interfacial Tension Measurements }}$}

Surface tension is the result of incomplete bonding of the molecules at the surface of a liquid leading to organized tangential stresses that compensate for the unbalanced normal stress. Such is the case of organic liquid surfaces in contact with air or any other gas. When the liquid surfaces contact another insoluble liquid, more adhesion forces are established across the interface between the molecules of the two different liquids. Thus, the interfacial tension (IFT) values are always lower than surface tension values (SFT). Thus, the IFT between two immiscible liquids is lower than the corresponding SFT values of each liquid.

We measured the IFT values of the samples listed in Table 2-1. The results from all measurements are reported in Table 3-1. In each measurement, the aqueous phase, the solvent ID, the ambient temperature, the identity of the du Nouy ring used (A, B, C, or D), the measured interfacial surface tension, the absolute (or temperature corrected) surface tension, the average of the duplicate measurements, the STDEV, and the \% RSD are reported.

At first, a visual inspection of the interfacial tension between NGS and the salt simulant appears to be constant in the range of samples tested. The values ranged from 15 to 18 dynes $/ \mathrm{cm}$. These values are consistent with the values observed when testing a similar composition of NG-CSSX solvent and with a similar salt simulant ( $\sim 15$ to 16$).{ }^{12}$ These values are also similar to the values observed when testing NG-CSSX with similar salt solution and boric acid. ${ }^{13}$ Likewise, the interfacial tension between NGS and $10 \mathrm{mM}$ boric acid were approximately the same (from 12 to 15 dynes/cm) compared to NGCSSX and $10 \mathrm{mM}$ boric acid (13.6 dynes/cm). ${ }^{12}$ These differences are expected since there is a compositional difference between CSSX and NGS and some of the chemicals in NGS are different from those in CSSX. The lower interfacial tensions values are significantly higher than the minimum interfacial values recommended for the NGCSSX/boric acid interface $\left(>5\right.$ dynes/cm). ${ }^{14}$ The lower interfacial values did not affect 
droplet formation and coalescing in the recent V-5/V-10 centrifuge testing between NGS and salt simulant. ${ }^{5}$

A statistical analysis was conducted to assess the impact of the chemical components in the solvent upon the static interfacial tension. The mean line (blue line) is within the confidence lines in Figure 3-1. However, a closer inspection reveals that both the modifier and the MaxCalix, together, affect the interfacial tension more statistically significantly than the Isopar $^{\circledR}$ L. ${ }^{\text {viii }}$ Their combined effect is to increase the static interfacial tension as shown in Figure 3-1. No other components affected the interfacial tension to the same extent. Fig. 3-1 only shows those variables of the fitting polynomial that are statistically significant. Note when reading Fig. 3-1, terms with $r^{2}>0.75$ are considered to significantly affect the interfacial tension (IFT). This polynomial fitting is by no means meant to predict future IFT values. The analysis instead shows which chemical constituent(s) of the organic blend affects the IFT values. In immiscible liquidliquid processes, chemical variations of the liquids due to evaporation, adsorption, decomposition, dilution or partitioning routinely occur and those variations in turn affect the physical properties of the liquids. Relating these chemical changes to established or developed models for predicting the physical properties of liquids is discussed later in this paper.

In the case of solvent contacting boric acid, TiDG significantly affected the measured static surface tension (see Figure 3-2). As shown in Figure 3-2, increasing the concentration of TiDG lowers the static interfacial tension. This may be explained by the fact that neutral TiDG protonates in contact with dilute boric acid (see Eq. 1).

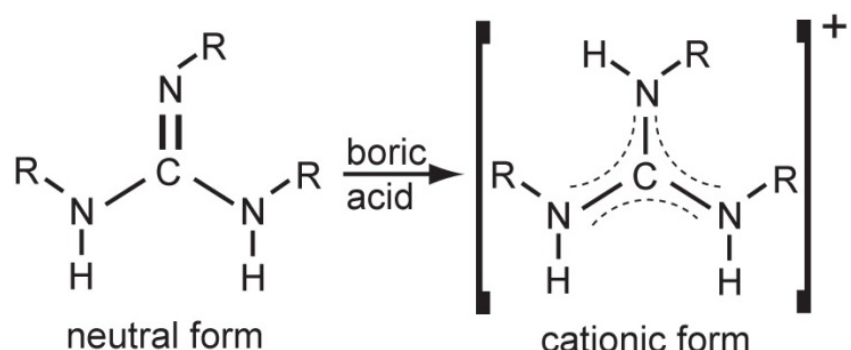

EQ. 1

\footnotetext{
viii If the $95 \%$ confidence curve (the two hyperbolic lines) do not include the mean value (broken blue line in the graph), the variable (X-axis) does affect the measured property.
} 
SRNL-STI-2013-00733

Revision 0

Table 3-1. Results from Interfacial Tension (IFT) Measurements

\begin{tabular}{|c|c|c|c|c|c|c|c|c|}
\hline & & & & Measured & Absolute & Average & & \\
\hline Aqueous Phase & Solvent ID & Temp. ${ }^{\circ} \mathrm{C}$ & Ring & dynes/cm & dynes/cm & dynes/cm & STDEV & $\%$ RSD \\
\hline \multirow{2}{*}{ boric acid } & NGS-1 & 22.5 & A & 14.9 & 14.126 & & & \\
\hline & & & & & & 14.591 & 0.657204 & $4.50 \%$ \\
\hline boric acid & NGS-1 & 22.5 & B & 15.8 & 15.056 & & & \\
\hline \multirow[t]{2}{*}{ V-5/10 salt simulant } & NGS-1 & 22.5 & C & 18.2 & 16.419 & & & \\
\hline & & & & & & 16.896 & 0.6747 & $3.99 \%$ \\
\hline V-5/10 salt simulant & NGS-1 & 22.5 & $\mathrm{D}$ & 19.2 & 17.373 & & & \\
\hline \multirow[t]{2}{*}{ boric acid } & NGS-2 & 21.0 & $\mathrm{D}$ & 14.3 & 13.560 & & & \\
\hline & & & & & & 12.439 & 1.584505 & $12.74 \%$ \\
\hline boric acid & NGS-2 & 21.0 & $\mathrm{~A}$ & 12.1 & 11.319 & & & \\
\hline \multirow[t]{2}{*}{ V-5/10 salt simulant } & NGS-2 & 21.0 & $B$ & 17.8 & 16.054 & & & \\
\hline & & & & & & 15.013 & 1.471702 & $9.80 \%$ \\
\hline V-5/10 salt simulant & NGS-2 & 21.0 & $\mathrm{C}$ & 15.6 & 13.972 & & & \\
\hline \multirow[t]{2}{*}{ boric acid } & NGS-5 & 20.5 & C & 13.8 & 13.157 & & & \\
\hline & & & & & & 13.365 & 0.29399 & $2.20 \%$ \\
\hline boric acid & NGS-5 & 20.5 & $\mathrm{~B}$ & 14.2 & 13.573 & & & \\
\hline \multirow[t]{2}{*}{ V-5/10 salt simulant } & NGS-5 & 20.5 & $\mathrm{D}$ & 18.9 & 17.140 & & & \\
\hline & & & & & & 17.140 & 0 & $0.00 \%$ \\
\hline V-5/10 salt simulant & NGS-5 & 20.5 & $\mathrm{~B}$ & 18.9 & 17.140 & & & \\
\hline \multirow[t]{2}{*}{ boric acid } & NGS-6 & 20.5 & C & 15.9 & 15.721 & & & \\
\hline & & & & & & 15.339 & 0.539868 & $3.52 \%$ \\
\hline boric acid & NGS-6 & 20.5 & $\mathrm{~A}$ & 15.2 & 14.957 & & & \\
\hline \multirow[t]{2}{*}{ V-5/10 salt simulant } & NGS-6 & 20.5 & C & 17.9 & 16.257 & & & \\
\hline & & & & & & 16.642 & 0.545449 & $3.28 \%$ \\
\hline V-5/10 salt simulant & NGS-6 & 20.5 & $\mathrm{D}$ & 18.7 & 17.028 & & & \\
\hline \multirow[t]{2}{*}{ boric acid } & NGS-7 & 20.5 & $B$ & 15.5 & 15.070 & & & \\
\hline & & & & & & 14.857 & 0.30152 & $2.03 \%$ \\
\hline boric acid & NGS-7 & 20.5 & $\mathrm{~A}$ & 15.1 & 14.644 & & & \\
\hline \multirow[t]{2}{*}{ V-5/10 salt simulant } & NGS-7 & 20.5 & $B$ & 17.9 & 16.213 & & & \\
\hline & & & & & & 16.453 & 0.339162 & $2.06 \%$ \\
\hline $\mathrm{V}-5 / 10$ salt simulant & NGS-7 & 20.5 & $\mathrm{D}$ & 18.4 & 16.693 & & & \\
\hline \multirow[t]{2}{*}{ boric acid } & NGS-11 & 20.5 & C & 13.5 & 13.137 & & & \\
\hline & & & & & & 12.976 & 0.226475 & $1.75 \%$ \\
\hline boric acid & NGS-11 & 20.5 & C & 13.2 & 12.816 & & & \\
\hline V-5/10 salt simulant & NGS-11 & 20.5 & $\mathrm{D}$ & 17.3 & 15.683 & & & \\
\hline & & & & & & 15.443 & 0.338709 & $2.19 \%$ \\
\hline $\mathrm{V}-5 / 10$ salt simulant & NGS-11 & 20.5 & $\mathrm{~B}$ & 16.8 & 15.204 & & & \\
\hline boric acid & NGS-13 & 20.0 & $\mathrm{D}$ & 13.8 & 13.307 & & & \\
\hline & & & & & & 13.466 & 0.224171 & $1.66 \%$ \\
\hline boric acid & NGS-13 & 20.0 & $\mathrm{C}$ & 14.1 & 13.624 & & & \\
\hline V-5/10 salt simulant & NGS-13 & 20.0 & $B$ & 18.5 & 16.800 & & & \\
\hline & & & & & & 17.089 & 0.408769 & $2.39 \%$ \\
\hline V-5/10 salt simulant & NGS-13 & 20.0 & $\mathrm{~A}$ & 19.1 & 17.378 & & & \\
\hline boric acid & NGS-18 & 20.0 & $\mathrm{D}$ & 15 & 14.506 & & & \\
\hline & & & & & & 14.189 & 0.448286 & $3.16 \%$ \\
\hline boric acid & NGS-18 & 20.0 & $\mathrm{~B}$ & 14.4 & 13.872 & & & \\
\hline V-5/10 salt simulant & NGS-18 & 20.0 & $\mathrm{C}$ & 18.6 & 16.877 & & & \\
\hline & & & & & & 17.118 & 0.340128 & $1.99 \%$ \\
\hline V-5/10 salt simulant & NGS-18 & 20.0 & $A$ & 19.1 & 17.358 & & & \\
\hline
\end{tabular}


Presumably, the ionized TiDG accumulates at the interface, thickens the interface, and lowers the tension. Because of this effect, there may be a finite concentration of ionized TiDG molecules on the aqueous side of the interface. This ionization effect is stronger than the dispersion and polar forces the modifier, extractant, and Isopar ${ }^{\circledR} \mathrm{L}$ may bring at the interface with an aqueous solutions. This data is consistent with the observation that ionized TiDG is slightly soluble in $10 \mathrm{mM}$ boric acid.
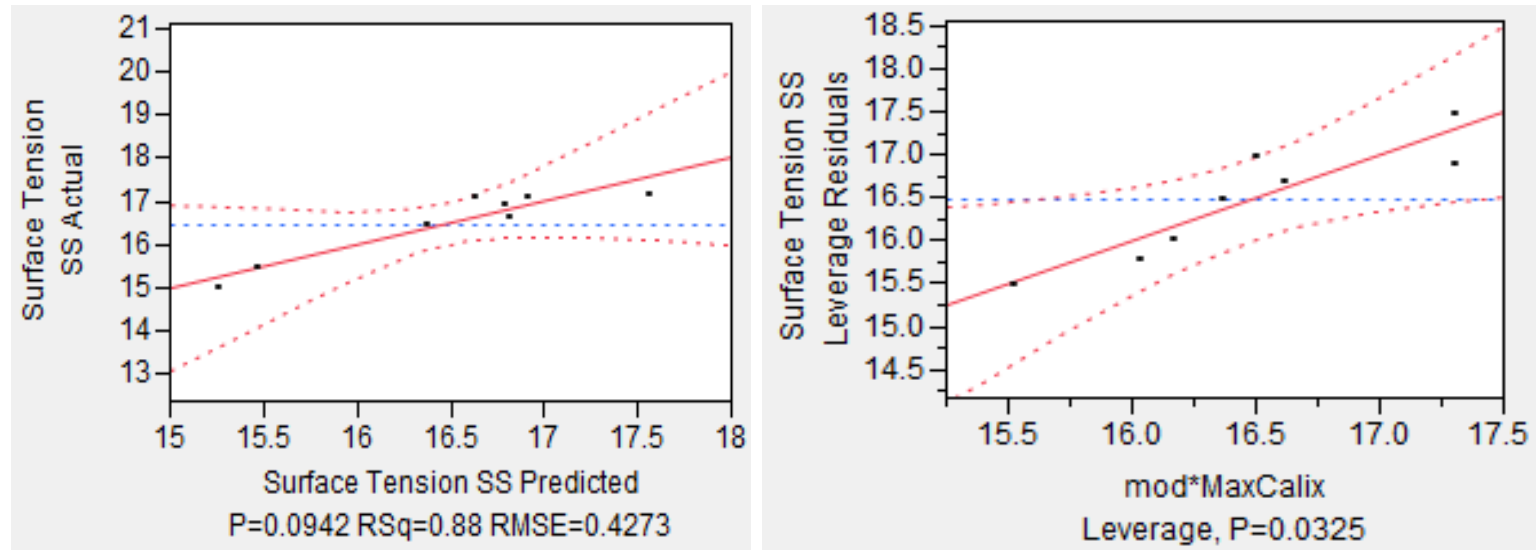

Figure 3-1. Influence of Modifier and MaxCalix on the interfacial tension between NGS and Salt Simulant
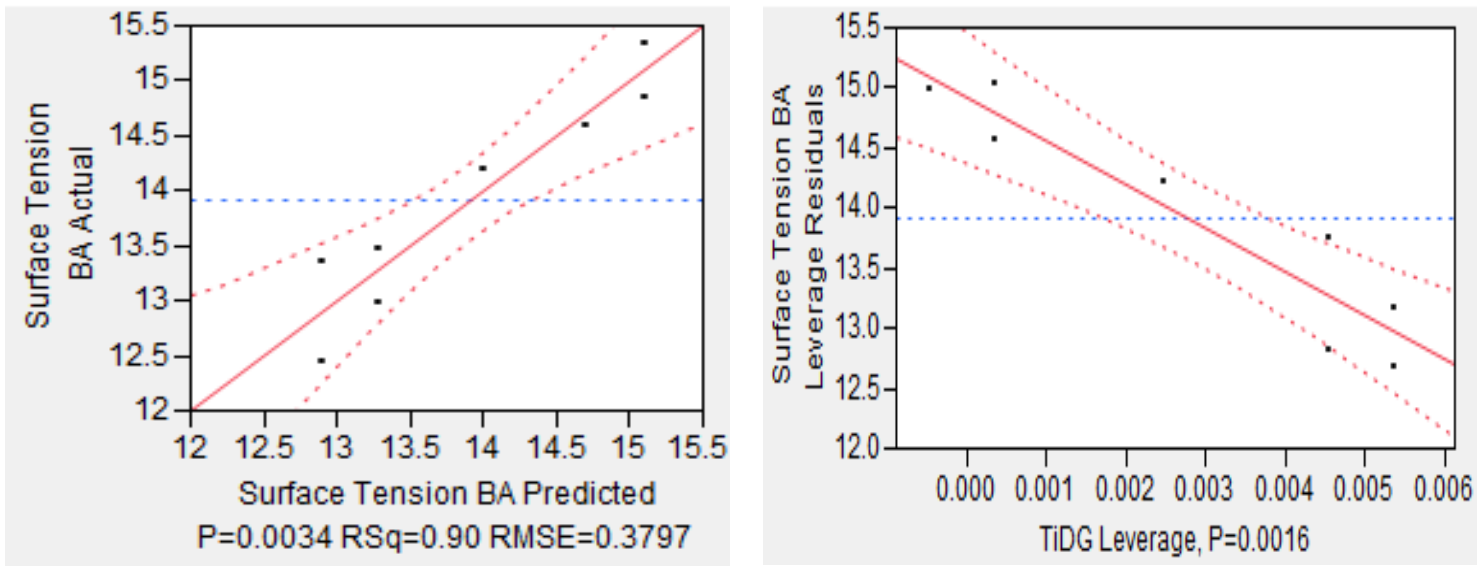

Figure 3-2. Influence of TiDG on the static interfacial tension between NGS and Boric acid 


\subsection{Comparison to Current Interfacial Tension Models (IFT)}

Fitting a polynomial function to the IFT data in Table 3-1 revealed the solvent's component that affected the IFT data the most. That fitting exercise, however, did not shed a light on the possible mechanisms of adsorption and surface interactions that occurs when two multicomponent fluids touch or form an interface. To that end, several adsorption theories were visited and applied to the data in Table 3-1. Theories that can fit the data with the least number of parameters and least number of mathematical operations were considered as plausible representation of the physical-chemical interactions that occur at the NGS-salt simulant and NGS-boric acid interfaces. Among the criteria used to select a model, the magnitude of any estimated parameters must be reasonable and consistent with the parameters of other similar systems; systems such as the Conoco (C12-C14) paraffin-tributyl phosphate/nitric acid system for which there is an extensive IFT dataset for comparison.

The IFT models considered fall into two categories: those based on a diffuse boundary (a finite miscibility between two liquids), and those based on a sharp interface. Since both solvent and aqueous carry over is observed with the centrifugal contactors at MCU and given the finite solubility of the modifier and TiDG in aqueous solutions, models based on a diffused interface cannot be neglected. These models rely on having knowledge of the components SFT values as well as their mutual solubility (convenient to the lab practitioner who can measure them readily). There is, however, one model that directly ties the molecular framework (or the chemistry of the substance) to the expected SFT and IFT values of two immiscible liquids. Using the group contribution method recommended by Hansen, ${ }^{15}$ the surface tension values for each of the NG-CSSX components was estimated. These values are listed in Table 3-2. The calculated value for Isopar ${ }^{\circledR} \mathrm{L}$ is $23.9 \mathrm{dyn} / \mathrm{cm}$. The literature cites a values of 24.6 (Exxon-Mobil MSDS April 2001) and $25.1 \mathrm{dyn} / \mathrm{cm}$ (Exxon-Mobil MSDS March 2002). The Isopar ${ }^{\circledR}$ L SFT value measured in this work was $21.91 \mathrm{dyn} / \mathrm{cm}$. The lower measured values might be the influence of a contaminated sample rather than an inadequacy of the model or a lower bias in the measurement. The SFT values of liquids typically range from 72 (water) to near zero dyn $/ \mathrm{cm}$. This range of values is too small to use IFT measurements as method for chemical identification. Because IFT values are always lower than SFT values, it is difficult to determine the chemical composition of a NG-CSSX solvent based on surface tension measurements. 
Table 3-2. The Hansen's solubility parameters for the NG-CSSX components and corresponding SFT

\begin{tabular}{|c|c|c|c|c|}
\hline & $\begin{array}{c}\text { Dispersion } \\
\left(\mathrm{MPa}^{1 / 2}\right)\end{array}$ & $\begin{array}{c}\text { Polar } \\
\left(\mathrm{MPa}^{1 / 2}\right)\end{array}$ & $\begin{array}{c}\text { Hydrogen } \\
\text { bonding } \\
\left(\mathrm{MPa}^{1 / 2}\right)\end{array}$ & $\begin{array}{c}\text { Predicted } \\
\text { dyn/cm }\end{array}$ \\
\hline TiDG & 6.7 & -0.5 & 0.3 & 26.1 \\
\hline Isopar $^{\circledR} \mathrm{L}$ & 7.4 & -0.1 & 0.4 & 23.9 \\
\hline Modifier & 8.3 & 6.9 & 5.6 & 55.7 \\
\hline MaxCalix & 11.1 & -1.9 & 3.5 & 91.6 \\
\hline
\end{tabular}

Hansen's solubility parameters are directly related to the enthalpy interaction parameters $(\chi)$ used in polymer solubility calculations $\left(\chi=\left(\delta_{\text {polymer }}-\delta_{\text {solvent }}\right)^{2} / \mathrm{RT}\right){ }^{16}$ Thus, group contribution theory can be used to estimate the IFT of a NG-CSSX solvent by knowing the concentration of the solvent components as shown in Table 3-3. Note that the predicted surface tensions in the last column of Table 3-3 are very similar to the surface tension measurements conducted at $\mathrm{ORNL}^{12}$ on NGS of similar composition to the samples studied here.

Table 3-3. The Hansen's solubility parameters and the predicted SFT estimation of the NGS samples

\begin{tabular}{|c|c|c|c|c|c|}
\hline Sample & $\begin{array}{c}\text { Molar } \\
\text { Volume } \\
\mathrm{mL} / \mathrm{mol}\end{array}$ & $\begin{array}{c}\text { Dispersion } \\
\left(\mathrm{MPa}^{1 / 2}\right)\end{array}$ & $\begin{array}{c}\text { Polar } \\
\left(\mathrm{MPa}^{1 / 2}\right)\end{array}$ & $\begin{array}{c}\text { Hydrogen } \\
\text { Bonding } \\
\left(\mathrm{MPa}^{1 / 2}\right)\end{array}$ & $\begin{array}{c}\text { Predicted } \\
\text { dyn/cm }\end{array}$ \\
\hline NGS-1 & 226.4 & 7.5 & 0.7 & 1.0 & 25.1 \\
\hline NGS-2 & 226.5 & 7.5 & 0.7 & 1.0 & 25.1 \\
\hline NGS-5 & 228.4 & 7.6 & 0.7 & 1.0 & 25.4 \\
\hline NGS-6 & 231.3 & 7.6 & 1.1 & 1.3 & 26.3 \\
\hline NGS-7 & 229.4 & 7.6 & 1.1 & 1.3 & 25.9 \\
\hline NGS-11 & 231.5 & 7.6 & 1.1 & 1.3 & 26.3 \\
\hline NGS-13 & 229.5 & 7.6 & 1.1 & 1.3 & 25.9 \\
\hline NGS-18 & 228.9 & 7.6 & 0.9 & 1.2 & 25.7 \\
\hline
\end{tabular}

The calculated SFT values listed in Table 3-3 can be inserted into IFT models to predict the IFT values of organic liquids. The computed IFT values are then compared to the measured IFT values shown in Table 3-1. The results of that effort to select the best predictive IFT model for the NG-CSSX solvent are shown in Section 3.2.

A literature survey revealed that most multicomponent IFT models consider binary mixtures. To apply these models to NGS, the NGS solvent was assumed to be a binary mixture of Isopar ${ }^{\circledR}$ L-modifier or Isopar ${ }^{\circledR}$ L-TiDG or Isopar ${ }^{\circledR}$ L-MaxCalix, or modifierTiDG. Models that make references to critical pressures or temperatures were not 
considered in this paper since such variables are not known for the extractant or modifier used in this work.

Borrowing from adhesion theory, the IFT value of two immiscible liquids is expected to be less than the SFT value of each liquid at the interface.

As an initial guide, adding one drop of immiscible liquid on another result in a partially wetted droplet. From adhesion theory, adding a droplet to a surface leads to the Young's equation of partial wetting.

$$
\sigma_{s v}=\sigma_{l s}+\sigma_{l v} \cos \theta
$$

For two miscible liquids, the wetting angle is larger than 90 . Thus, $\cos (\theta)$ is approximately -1 and therefore, $\sigma_{1 \mathrm{v}}-\sigma_{2 \mathrm{v}}=\sigma_{12}$. For example, the SFT of water and ndodecane is $72.7 \mathrm{dyn} / \mathrm{cm}$ and $25 \mathrm{dyn} / \mathrm{cm}$ respectively. ${ }^{17}$ The IFT of dodecane on water based on Eq.2 should be $47.7 \mathrm{dyn} / \mathrm{cm}$ and measurements indicate a value of approximately $52 \mathrm{dyn} / \mathrm{cm}$. Thus, we expect the $\mathrm{IFT}_{12}$ (between two immiscible liquids) to be near the difference of the SFT of the individual components. If the individual liquids have hydrogen-bonding capable constituents, their IFT value will be much lower than predicted from Eq. 2.

To strengthen the prediction capabilities of Eq. 2, terms have been added to Eq. 2 to account for adhesion forces across the interface between two immiscible liquids as shown in Eq. 3.

$$
\gamma_{\mathrm{s} 1}=\gamma_{\mathrm{lv}}+\gamma_{\mathrm{sv}}-2 \sqrt{\gamma_{\mathrm{lv}} \gamma_{\mathrm{sv}}} e^{-\beta\left(\gamma_{\mathrm{lv}}-\gamma_{\mathrm{sv}}\right)^{2}}
$$

The last term in the right hand side of Eq. 3 includes the geometric mean of the liquids SFT values and an exponential term that includes the square difference of the liquids SFT values. ${ }^{18}$ The data in Table 3-1 was fitted with Eq. 3. The results are shown in Table 3-4.

\begin{tabular}{|c|c|c|c|c|}
\hline Solution & \multicolumn{2}{|c|}{ Boric Acid } & \multicolumn{2}{|c|}{ Salt Simulant } \\
\hline & Isopar $^{\circledR}$ L/Modifier & $\begin{array}{c}\text { Isopar }^{\mathbb{Q}} \mathrm{L} \\
\text { /TiDG }^{\text {Th }}\end{array}$ & $\begin{array}{c}\text { Isopar }^{\mathbb{R}} \mathrm{L} \\
/ \mathrm{mod}\end{array}$ & $\begin{array}{c}\text { Isopar }^{\mathbb{R}} \mathrm{L} \\
\text { /TiDG }^{\text {To }}\end{array}$ \\
\hline Parameter: $\beta$ & $(1.05 \pm 0.15) \mathrm{E}-03$ & $\begin{array}{c}(6.3 \pm 0.5) \\
\text { E-03 }\end{array}$ & $\begin{array}{l}\text { Failed to } \\
\text { fit }\end{array}$ & $\begin{array}{c}(9.03 \pm 0.5) \\
\text { E-02 }\end{array}$ \\
\hline $\begin{array}{l}\text { Replacing } \\
\text { the } \\
\text { exponential } \\
\text { term in EQ. } \\
3 \text { with a } \\
\text { constant }\end{array}$ & $(1.4 \pm 0.02) \mathrm{E}-02$ & $\begin{array}{c}(2 \pm 0.06) \\
\mathrm{E}-02\end{array}$ & $0.86 \pm 0.01$ & $0.672 \pm 0.02$ \\
\hline
\end{tabular}

Table 3-4. Parameters values from Eq. 3 that best fit the data in Table 3-1 
Table 3-4 shows the best value of the parameter in Eq. 3 that fitted the data in Table 3-1 assuming the solvents are binary mixtures of either Isopar ${ }^{\circledR} \mathrm{L}$ and modifier or Isopar ${ }^{\circledR} \mathrm{L}$ and TiDG in contact with boric acid or salt stimulant. Equation 3 failed to fit the data under one of the assumptions (Isopar ${ }^{\circledR}$ L-modifier in contact with salt stimulant). A better and more consistent fit was obtained when the exponential term in Eq. 3 was replaced with a constant. The fourth row in Table 3-4 shows the value of a parameter with less uncertainty and more stable.

Another empirical SFT model is the Parachor's model. ${ }^{19}$ In this model, the surface tension (SFT) is proportional to the organic liquid density raised to the fourth power (see Eq. $3 b)$.

$$
\sigma^{1 / 4}=\rho_{\mathrm{M}}^{\mathrm{L}} \sum x_{i} P_{i}
$$

EQ. 3B

In this equation, $\rho, \sigma, x_{i}, P_{i}$ stands for molar density of the organic liquid, surface tension, mole fraction of the $i^{\text {th }}$ constituent in the organic liquid, and the Parachor parameter for each constituent in the organic liquid. This model fitted the IFT of NGS in salt simulant and in boric acid. The Parachor constants found were 3.4, 2.9, 1.6, and 1.3 for MaxCalix, modifier, Isopar ${ }^{\circledR} \mathrm{L}$, and TiDG respectively. The constant varied for the case of NGS contacting boric acid. The Parachor constant for this case were 2.4, 1.9, 1.3, 0.9 respectively. Because the parameter varies with the aqueous solution type, this method is aqueous solution specific. Chemical variations of the NGS appear to have minimal impact on the values of these parameters.

Considering the NG-CSSX solvent as a two component system (Isopar ${ }^{\circledR} \mathrm{L}$ as one component and the modifier-suppressor-extractant combo - i.e., the "balance of the solvent" - as the other component), the ideal equation shown in Eq. 4, developed by Wilson $^{20}$, can be applied to determine its predictability with the NG-CSSX solvent. This model includes deviations from the ideal additive mixture law $\left(\mathrm{x}_{1} \sigma_{1}+\mathrm{x}_{2} \sigma_{2}\right)$ that arises when components interact with each other. The Wilson model was applied by using the measured IFT value of $24 \mathrm{dyn} / \mathrm{cm}$ for Isopar ${ }^{\circledR} \mathrm{L}$ and the calculated value of $56 \mathrm{dyn} / \mathrm{cm}$ (as calculated in Table 3-2) for the "balance of the solvent" to estimate the coefficients of interactions $\left(f_{12}\right.$ and $\left.f_{21}\right)$ shown in Eq. 4 . The model fit the data well (maximum error found was $10 \%$ of measurement) when the Isopar ${ }^{\circledR} \mathrm{L}$ and balance of solvent IFT's take the values shown in Table 3-5 for the NG-CSSX contacting salt simulant (SS) and Table 3-6 for NG-CSSX contacting $10 \mathrm{mM}$ boric acid (BA). With this model, the interaction parameters (the degree to which one component affect another component at the interface) were $f_{12}=0.08$ and $f_{21}=1.04$ when the solvent contacted salt simulant and 14 and 1.04 when the solvent contacted boric acid.

$$
\sigma_{\text {mixture }}=\frac{x_{1} \sigma_{1}}{x_{1}+f_{12} x_{2}}+\frac{x_{2} \sigma_{2}}{x_{2}+f_{21} x_{1}}-\frac{x_{1} x_{2}\left|\sigma_{1}-\sigma_{2}\right|}{\left(x_{1}+f_{12} x_{2}\right)\left(x_{1}+f_{12} x_{2}\right)} \quad \text { EQ. } 4
$$


Where $f_{12}=\frac{A_{2}}{A_{1}} e^{-\left[\left(g_{12}-g_{11}\right) / R T\right]}, x_{i}$ stands for the mole fraction,

and $A_{i}$ stands for the molecular surface area of component " $i "$

Table 3-5. Fitting results of the NG-CSSX/Salt Simulant with Wilson's model.

$f_{12}=0.08$ and $f_{21}=1.04$. All units given as $\mathrm{dyn} / \mathrm{cm}$.

\begin{tabular}{|c|c|c|c|c|c|}
\hline Solvent & Isopar ${ }^{\circledR}$ L & $\begin{array}{c}\text { Balance } \\
\text { of } \\
\text { Solvent* }\end{array}$ & EQ. 4 & $\begin{array}{c}\text { NG-CSSX/ } \\
\text { Salt } \\
\text { Simulant }\end{array}$ & $\begin{array}{c}\text { Residual } \\
\text { (Measured - } \\
\text { EQ. 4) }\end{array}$ \\
\hline NGS-1 & 24.0 & 56.0 & 16.48 & 16.90 & 0.42 \\
\hline NGS-2 & 24.0 & 56.0 & 16.49 & 15.01 & -1.48 \\
\hline NGS-5 & 24.0 & 56.0 & 16.49 & 17.14 & 0.65 \\
\hline NGS-6 & 24.0 & 56.0 & 16.50 & 16.64 & 0.14 \\
\hline NGS-7 & 24.0 & 56.0 & 16.51 & 16.45 & -0.06 \\
\hline NGS-11 & 24.0 & 56.0 & 16.51 & 15.44 & -1.07 \\
\hline NGS-13 & 24.0 & 56.0 & 16.51 & 17.09 & 0.58 \\
\hline NGS-18 & 24.0 & 56.0 & 16.32 & 17.12 & 0.80 \\
\hline
\end{tabular}

*Balance of solvent includes modifier-suppressor-extractant combo

Table 3-6. Fitting results of the NG-CSSX/Boric Acid with Wilson's Model.

$f_{12}=14$ and $f_{21}=1.04$. All units given as $\mathrm{dyn} / \mathrm{cm}$.

\begin{tabular}{|c|c|c|c|c|c|}
\hline Sample & Isopar ${ }^{\circledR}$ L & $\begin{array}{c}\text { Balance } \\
\text { of } \\
\text { Solvent* }\end{array}$ & EQ. 4 & $\begin{array}{c}\text { NG-CSSX/ } \\
\text { Boric Acid }\end{array}$ & $\begin{array}{c}\text { Residual } \\
\text { (Measured - } \\
\text { EQ. 4) }\end{array}$ \\
\hline NGS-1 & 24.0 & 56.0 & 13.37 & 14.591 & 1.22 \\
\hline NGS-2 & 24.0 & 56.0 & 13.37 & 12.439 & -0.93 \\
\hline NGS-5 & 24.0 & 56.0 & 13.37 & 13.365 & 0.00 \\
\hline NGS-6 & 24.0 & 56.0 & 13.99 & 15.339 & 1.35 \\
\hline NGS-7 & 24.0 & 56.0 & 14.00 & 14.857 & 0.86 \\
\hline NGS-11 & 24.0 & 56.0 & 13.99 & 12.976 & -1.01 \\
\hline NGS-13 & 24.0 & 56.0 & 14.00 & 13.466 & -0.53 \\
\hline NGS-18 & 24.0 & 56.0 & 13.51 & 14.189 & 0.68 \\
\hline
\end{tabular}

*Balance of Solvent includes modifier-suppressor-extractant combo

The asymmetry $\mathrm{f}_{21}=14$ could mean a strong Isopar ${ }^{\circledR} \mathrm{L}$-modifier interaction when the solvent contacts dilute boric acid. 
Another model similar to Wilson's model that fitted the data well was the HildebrandScott equation (EQ. 5). ${ }^{21}$

$$
\sigma_{\mathrm{m}}=y_{1} \sigma_{1}+y_{2} \sigma_{2}-\frac{\left(\sigma_{1}-\sigma_{2}\right)^{2}}{2 R T}\left(y_{1} A_{2}+y_{2} A_{1}\right) y_{1} y_{2}
$$

Where the "T", $A_{1}$, and $A_{2}$ stand for temperature (Kelvin), and the two other constants stand for molar area. Table 3-7 shows the fitting results for NG-CSSX in salt simulant. This equation fitted the data in Table 3-1 for boric acid only when the constant " $\mathrm{A}_{1}$ " is set equal to zero (sub-index one is assigned to Isopar ${ }^{\circledR} \mathrm{L}$ and sub-index two is assigned to the "Balance of Solvent") as shown in Table 38.

Table 3-7. Prediction performance of the Hildebrand-Scott model for NG-CSSX/Salt simulant (all units in dyn/cm). $A_{2}=27.5$ and $A_{1}=32$.

\begin{tabular}{|c|c|c|c|c|}
\hline Solvent & Isopar $^{\circledR}$ L & $\begin{array}{c}\text { Balance } \\
\text { of } \\
\text { Solvent* }\end{array}$ & Eq. 5 & $\begin{array}{c}\text { NG- } \\
\text { CSSX/ } \\
\text { Salt } \\
\text { Simulant }\end{array}$ \\
\hline NGS-1 & 23.6 & 56.7 & 17.37 & 16.90 \\
\hline NGS-2 & 22.8 & 58.0 & 15.56 & 15.01 \\
\hline NGS-5 & 23.6 & 56.4 & 16.27 & 17.14 \\
\hline NGS-6 & 24.1 & 54.9 & 17.47 & 16.64 \\
\hline NGS-7 & 24.2 & 55.1 & 17.10 & 16.45 \\
\hline NGS-11 & 23.9 & 56.2 & 15.43 & 15.44 \\
\hline NGS-13 & 24.4 & 54.7 & 15.91 & 17.09 \\
\hline NGS-18 & 24.1 & 55.6 & 16.82 & 17.12 \\
\hline
\end{tabular}

*Balance of Solvent includes modifier-extractant-suppressor

Table 3-8. Prediction performance of the Hildebrand-Scott model for NG-CSSX/Boric Acid (all units in dyn/cm). $A_{2}=40$ and $A_{1}=0$.

\begin{tabular}{|c|c|c|c|c|}
\hline Solvent & Isopar $^{\circledR}$ L & $\begin{array}{c}\text { Balance } \\
\text { of } \\
\text { Solvent* }\end{array}$ & Eq. 5 & $\begin{array}{c}\text { NG- } \\
\text { CSSX/Boric } \\
\text { Acid }\end{array}$ \\
\hline NGS-1 & 23.8 & 56.4 & 14.59 & 14.08 \\
\hline NGS-2 & 23.0 & 57.7 & 12.44 & 12.63 \\
\hline NGS-5 & 23.3 & 57.2 & 13.37 & 13.00 \\
\hline NGS-6 & 24.5 & 54.5 & 15.34 & 14.89 \\
\hline NGS-7 & 24.4 & 54.7 & 14.86 & 14.11 \\
\hline NGS-11 & 23.9 & 56.2 & 12.98 & 12.44 \\
\hline NGS-13 & 24.1 & 55.7 & 13.47 & 13.21 \\
\hline NGS-18 & 24.0 & 55.9 & 14.19 & 14.70 \\
\hline
\end{tabular}

*Balance of Solvent includes modifier-extractant-suppressor 
Yet another thermodynamically derived IFT model based on the finite and small exchange of components between two immiscible liquids has been proposed by Jufu. ${ }^{20}$ In this model the solubility of the transferrable components from one liquid is small in the other liquid and vice-versa. This is equivalent to the solubility of cesium nitrate in NG-CSSX and the solubility of the modifier or TiDG in the aqueous phase. The derived equation (EQ 6) predicts large mole fractions $(>0.28)$ for the transferable component that will give surface tension comparable to those measured in this work.

$$
\sigma^{\prime}=\frac{K R T X}{A_{\mathrm{w} 0} \exp (X)\left(x_{1}^{\prime \prime} q_{1}+x_{2}^{\prime} q_{2}+x_{3 \mathrm{r}} q_{3}\right)}
$$

EQ. 6

where $\boldsymbol{X}=-\ln \left(x_{1}^{\prime \prime}+x_{2}^{\prime}+x_{3 \mathrm{r}}\right)$ and all the $x_{\mathrm{i}}$ stands for mole fractions and the symbols ,", and , ', stands for two immiscible liquids. With four fitting parameters (K/A, $\mathrm{q}_{1}, \mathrm{q}_{2}$, and $\mathrm{q}_{3}$ ), this model can only fit the values in Table 3-1 only with unreasonable solubility of NGS in salt simulant and vice versa as shown in Table 3-9.

Table 3-9. Mole fraction solubility of NGS in salt simulant and vice versa as predicted in Eq. 6

\begin{tabular}{|c|c|c|c|}
\hline $\begin{array}{c}\text { NGS-Salt } \\
\text { Simulant } \\
\text { (dyn/cm) }\end{array}$ & $\begin{array}{r}\text { Calculated IFT } \\
\mathbf{( d y n} / \mathbf{c m})\end{array}$ & $\begin{array}{r}\text { Salt Simulant in } \\
\text { NGS (mole fraction) }\end{array}$ & $\begin{array}{c}\text { NGS in Salt } \\
\text { Simulant } \\
\text { (mole fraction) }\end{array}$ \\
\hline 16.90 & 12.79 & 0.35 & 0.61 \\
\hline 15.01 & 15.01 & 0.29 & 0.66 \\
\hline 17.14 & 17.14 & 0.28 & 0.66 \\
\hline 16.64 & 16.64 & 0.28 & 0.66 \\
\hline 16.45 & 16.45 & 0.28 & 0.66 \\
\hline 15.44 & 15.44 & 0.29 & 0.66 \\
\hline 17.09 & 17.09 & 0.28 & 0.66 \\
\hline 17.12 & 17.12 & 0.28 & 0.66 \\
\hline
\end{tabular}

Szyzkowski ${ }^{22}$ developed an IFT equation for a binary mixture of organic liquids over water. That equation assumes that the relative rate of adsorption of the two components to the water interface follows a Langmuir isotherm and determines both the extent of adsorption and the equilibrium IFT. With those assumptions, EQ. 7 was derived.

$$
\sigma^{\text {id }}=\sigma_{2}^{0}-R T \Gamma_{\mathrm{m}} \ln \left\{1+\left(q_{12}-1\right) x_{1}\right\}
$$

In EQ. $7, \sigma_{2}$ is the SFT of the pure component "2", $\Gamma_{\mathrm{m}}$ stands for the surface concentration that leads to a monolayer formation, $q_{12}$ is the ratio of adsorption and desorption from the interface, and $x_{1}$ is the mole fraction of component " 1 " in the bulk organic liquid. Thus, adding component "1" or "1" adsorbs faster than component "2" decreases the interfacial tension from the pure component IFT. In our case, we treated Isopar $^{\mathbb{B}} \mathrm{L}$ as component "2" and added "Balance of Solvent" until the surface tension 
decreases from that of pure Isopar ${ }^{\circledR} \mathrm{L}(24 \mathrm{dyn} / \mathrm{cm})$ to either 16.9 (obtained from contacting salt simulant) or 14 (obtained from contacting boric acid). When the experimental IFT value was $16.9 \mathrm{dyn} / \mathrm{cm}, \mathrm{EQ} .7$ obtained this number when RT $\Gamma_{\mathrm{m}}, q_{12}$, and $x_{1}$ were 20,5 , and 0.11 respectively. The calculated $x_{1}$ is similar to the 0.11 mole fraction of "Balance of Solvent" in this solvent. Therefore, this equation not only can predict future NG-CSSX IFT values but it indicates an ideal behavior between $\operatorname{Isopar}^{\circledR} \mathrm{L}$ and the "Balance of Solvent".

A similar fitting success was obtained with the expression derived by Sowanane ${ }^{23}$ where non-ideal behavior is taken into account for the IFT between two immiscible solvents. The resulting equation has mathematical terms similar to that derived by Flory-Huggins (see Eq. 8).

$$
\sigma=\sigma_{\text {id }}+R T X_{1} X_{2}\left(1 / A_{1}-1 / A_{2}\right)\left\lfloor\delta_{\mathrm{p}}+\delta_{\mathrm{m}} X_{2}\right\rfloor \quad \text { EQ. } 8
$$

In Eq. $8, \mathrm{~A}_{1}, \mathrm{~A}_{2}, \delta_{\mathrm{p}}, \delta_{\mathrm{m}}$ are constant terms and the last two terms account for non-ideality in the interaction between components. Also in Eq. 8, $\sigma_{\text {ideal }}=X_{1} \sigma_{1}+X_{2} \sigma_{2}$. Because there are two fitting constants and the addition of a square mole fraction $\left(\mathrm{X}_{2}\right)$, this equation was able to fit the entire range of the data as shown in Table 3-10.

Table 3-10. The fitting performance from Eq. 8

\begin{tabular}{|c|c|c|}
\hline $\begin{array}{c}\text { Solvent } \\
\text { ID }\end{array}$ & $\begin{array}{c}\text { NGS/Salt } \\
\text { Solution } \\
\text { (dyn/cm) }\end{array}$ & $\begin{array}{c}\text { EQ 8 } \\
\text { (dyn/cm) }\end{array}$ \\
\hline NGS-1 & 16.90 & 16.90 \\
\hline NGS-2 & 15.01 & 16.85 \\
\hline NGS-5 & 17.14 & 16.33 \\
\hline NGS-6 & 16.64 & 15.95 \\
\hline NGS-7 & 16.45 & 16.45 \\
\hline NGS-11 & 15.44 & 15.89 \\
\hline NGS-13 & 17.09 & 16.40 \\
\hline NGS-18 & 17.12 & 16.39 \\
\hline
\end{tabular}

Another empirical model based on binary mixture is that proposed by Burgess. ${ }^{24}$ This model is based on the IFT of the individual constituents that make up an organic liquid. For a binary organic liquid, the model prediction is shown in Eq. 9.

$$
\gamma=\left(\gamma_{1}-\gamma_{2}\right) \exp \left(-\alpha V^{0.7}\right)+\gamma_{2}
$$

In Eq. 9, $\gamma_{1}, \gamma_{2}$, and $V$ stand for the IFT of component "1", component " 2 ", and the volume fraction of component " 2 " in the organic liquid respectively. The term " $\alpha$ " is a constant. Since we don't know the IFT of Isopar ${ }^{\circledR} L$ or the IFT of the "Balance of Solvent" relative to our salt simulant or boric acid, we used this equation to estimate those values. 
The best fitting of the data in Table 3-1 was obtained when $\alpha$ equaled 4.0. The estimated IFT values for Isopar ${ }^{\circledR} \mathrm{L}$ and the "Balance of Solvent" are shown in Table 3-11. From Table 3-11, the change from salt solution to boric acid selectively affected the "Balance of Solvent's" IFT value. Experimental IFT data of the "Balance of Solvent" and Isopar ${ }^{\circledR}$ L in contact with salt solution and boric acid is needed to verify the predictions shown in Table 3-9.

Table 3-11. Estimates of the IFT values of the "Balance of Solvent"and Isopar ${ }^{\circledR} L$ in Salt Solution and in Boric Acid using Eq. 9

\begin{tabular}{|c|c|c|c|c|c||}
\hline $\begin{array}{l}\text { NGS-SS } \\
(\text { dyn/cm })\end{array}$ & $\begin{array}{l}\text { IFT “Balance } \\
\text { of } \\
\text { Solvent”/SS } \\
(\text { dyn/cm })\end{array}$ & $\begin{array}{l}\text { IFT } \\
\text { Isopar }{ }^{\circledR} \text { L/SS } \\
(\text { dyn/cm })\end{array}$ & $\begin{array}{l}\text { NGS- } \\
\text { Boric } \\
\text { Acid } \\
(\text { dyn/cm })\end{array}$ & $\begin{array}{l}\text { IFT “Balance } \\
\text { of } \\
\text { Solvent”/BA } \\
(\text { dyn/cm })\end{array}$ & $\begin{array}{l}\text { IFT } \\
\text { Isopar } \\
(\text { dyn/cm })\end{array}$ \\
\hline 16.896 & 17.0 & 14.8 & 14.591 & 14.6 & 14.7 \\
\hline 15.013 & 15.0 & 13.9 & 12.439 & 12.4 & 13.9 \\
\hline 17.14 & 17.2 & 14.4 & 13.365 & 13.3 & 14.2 \\
\hline 16.642 & 16.7 & 15.0 & 15.339 & 15.4 & 14.9 \\
\hline 16.453 & 16.5 & 14.8 & 14.857 & 14.9 & 14.8 \\
\hline 15.443 & 15.5 & 14.1 & 12.976 & 12.9 & 14.0 \\
\hline 17.089 & 17.2 & 14.4 & 13.466 & 13.4 & 14.2 \\
\hline 17.118 & 17.2 & 14.6 & 14.189 & 14.2 & 14.5 \\
\hline
\end{tabular}

SS $=$ Salt Simulant

$\mathrm{BA}=$ Boric Acid

$\alpha$ equaled 4.0

Balance of Solvent includes the modifier-extractant-suppressor combo

Yet another empirical IFT model based on the partial miscibility between an organic and an aqueous solution influences the interfacial tension (IFT). ${ }^{25}$ This model relates the constituents bulk concentration to the IFT as shown in Eq. 10.

$$
\sigma^{\alpha \beta}=0.022 \frac{T \ln \left(X_{W(O)} / X_{W(W)}\right)}{\left(X_{O(W)}\right)^{2}-\left(X_{O(O)}\right)^{2}}
$$

EQ. 10

In Eq. 10, " $X_{i(j)}$ " stand for the mole fraction of the $\mathrm{i}^{\text {th }}$ component in the $\mathrm{j}^{\text {th }}$ phase (water or organic liquid or at the interface labeled here as " $\alpha \beta$ "). The term " $T$ " stands for temperature (Kelvin). The solubility of Isopar ${ }^{\circledR}$ L, the modifier, TiDG or MaxCalix was recently measured in boric acid or in salt solution. ${ }^{14}$ Those measured solubility differed from the estimated solubility using Eq. 10. The estimated solubility (from Eq. 10) that a constituent must have in salt solution and in a NGS liquid to have the IFT values listed in Table 3-1. Table 3-12 shows the calculated solubility NGS should have in the salt simulant if the assumptions used to derived Eq. 10 apply to our sample and testing conditions. 
SRNL-STI-2013-00733

Revision 0

Table 3-12. The estimated solubility of NGS in salt simulant based on Eq. 10

\begin{tabular}{|c|c|c|}
\hline $\begin{array}{c}\text { NGS-Salt } \\
\text { simulant } \\
\text { (dyn/cm) }\end{array}$ & $\begin{array}{c}\mathrm{X}_{\text {salt solution }} \\
\text { in NGS }\end{array}$ & $\begin{array}{c}\mathrm{X}_{\mathrm{NGS}} \text { in } \\
\text { Salt } \\
\text { Simulant }\end{array}$ \\
\hline 16.896 & 0.074 & 0.008 \\
\hline 15.013 & 0.099 & 0.002 \\
\hline 17.14 & 0.071 & 0.009 \\
\hline 16.642 & 0.077 & 0.007 \\
\hline 16.453 & 0.079 & 0.007 \\
\hline 15.443 & 0.093 & 0.003 \\
\hline 17.089 & 0.072 & 0.009 \\
\hline 17.118 & 0.072 & 0.009 \\
\hline
\end{tabular}

$\mathrm{X}_{\mathrm{i}}$ stands for mole fraction

An inspection of Table 3-12 shows that mole fraction of NGS (or one of its constituents) in the salt simulant must range from 0.002 to 0.01 to obtain the measured IFT value listed in Table 3-12. While this concentration level (NGS in salt simulant) is within the range of the solvent physical carryover observed in testing done in SRNL, ORNL or Parsons, it is far greater than measured soluble concentrations. Therefore, this model will not predict IFT values for the NGS-salt simulant or NGS-boric acid interfaces with confidence.

The literature review also identified another thermodynamically consistent model, the Gibbs-Frumkin model that is similar to the Szyzkowski ${ }^{22}$ model except that the adsorption model is Frumkin instead of Langmuir. The Frumkin model is the Langmuir model but with an extra term to account for neighboring molecules interactions at the interface. This model prediction for a single species absorbing at the interface is shown in Eq. 11 and 12.

$$
\begin{aligned}
& \gamma_{0}-\gamma=-\frac{2 R T}{\omega}\left[\ln (1-\theta)+a \theta^{2}\right] \\
& b\left[c\left(c+c_{2}\right)\right]^{1 / 2} f=\frac{\theta}{1-\theta} \exp (-2 a \theta)
\end{aligned}
$$

In Eq. 11 and 12, $\theta, c, c_{2}, T, w$, and $f$ stands for molar interfacial coverage, organic constituent bulk concentration, aqueous ion bulk concentration in the aqueous solution, the temperature (Kelvin), molar surface area, and activity coefficient of the absorbing ion respectively. The constants are represented by the symbols " $a$ " and " $b$ ". The symbol " $a$ " represents the degree of interaction between the molecules at the interface $(a=0$ implies no interaction). The symbol " $b$ " represents the absorption equilibrium constant for that 
species to the interface. Assuming " $f$ " is unity and " $C+C_{2}$ " is nearly " $C$ ", the output from Eq.12 is shown in Table 3-13. A quick examination of the data in Table 3-1 reveals those samples with no TiDG or MaxCalix have reduced IFT values. Thus, modeling TiDG or MaxCalix as surfactants with this model yielded an unreasonable zero interfacial coverage at low IFT values. This model best fitted the data when the modifier was considered a surfactant. Table 3-11 shows the predicted IFT values and corresponding surface coverage for the modifier. The data presented in Table 3-13 was generated assuming no interaction $(a=0)$ between the modifier molecules at the interface and the left hand side of Eq. 12 is replaced by the term " $b c$ " (assuming $c_{2}=0$ or no solubility in the aqueous phase and an activity coefficient value of one). If these predictions are correct, a large fraction of the interfacial composition is composed of modifier. The modifier molecule contains polar (ethers and hydroxyls) and hydrogen bonding (hydroxyl) components that can interact with structure forming water at the interface with the salt simulant. In addition, the modifier bulk concentration is high enough to populate the interface and maintain solubility in the bulk.

Table 3-13. Prediction of the reduced IFT values (Table 3-1) using Eq. 11 and Eq. 12 assuming the modifier is the surface active agent in contact with salt simulant.

\begin{tabular}{|c|c|c|c|c|}
\hline $\begin{array}{c}\text { Measured } \\
\gamma_{\mathrm{o}}^{\text {NGS }}-\gamma\end{array}$ & $\begin{array}{c}\text { Predicted } \\
\gamma_{\mathrm{o}}^{\text {NGS }}-\gamma\end{array}$ & $\begin{array}{c}\text { Measured }- \\
\text { Predicted }\end{array}$ & $\begin{array}{c}\text { Modifier Bulk } \\
\text { Concentration }\end{array}$ & $\begin{array}{c}\text { Surface } \\
\text { Coverage } \\
(\theta)\end{array}$ \\
\hline 8.20 & 8.15 & 0.05 & 0.50 & 0.76 \\
\hline 10.09 & 8.15 & 1.94 & 0.50 & 0.76 \\
\hline 8.26 & 8.15 & 0.11 & 0.50 & 0.76 \\
\hline 9.66 & 9.99 & -0.33 & 0.75 & 0.83 \\
\hline 9.45 & 9.99 & -0.54 & 0.75 & 0.83 \\
\hline 10.86 & 9.99 & 0.87 & 0.75 & 0.83 \\
\hline 8.81 & 9.99 & -1.18 & 0.75 & 0.83 \\
\hline 8.58 & 9.18 & -0.6 & 0.63 & 0.80 \\
\hline $\begin{array}{l}a=0 \\
b=6.36\end{array}$ \\
$w=5.7$
\end{tabular}

\section{2 "Restricted" Diffusivity Measurement: NGS droplets in Salt Simulant}

Operation at MCU relies on the formation of dispersions and separation of immiscible liquids. These processes are influenced by operational conditions (such as turbulent flow, residence time, ionic strength, $\mathrm{pH}$, and temperature) and the fluid's physical properties such as interfacial tension (IFT) and viscosity (of the dispersed and continuous phases). In multicomponent mixtures, the measured viscosity is an average value of the mobility in the mixtures. Traditional viscosity measurement does not provide the individual mobility of each of the components that make up a mixture. Some components in a 
mixture play no interfacial role in dispersion formation and break-up. This work measured the individual mobility (diffusivity) of the components that make up the NGSCSSX solvent.

Before discussing and analyzing diffusivity data, recall that there are two effects that may impact self-diffusion and thus complicate the data analysis. Droplet sizes smaller than the mean of the square displacement of the molecules inside the droplet will yield a lower diffusivity constant bias since the molecules will contact the interface before they complete their mean translational path. ${ }^{26}$ Thus, this gives a method for relating droplet size and diffusivity measurements by NMR. The opposite is also true. For large droplets, if the waiting time before reading the magnetization is too short relative to the time the molecules need to complete one mean squared displacement, the diffusivity constant will be biased low.

In addition to that effect, there is the chemical composition of the droplets that can also affect the diffusivity inside the droplets. Varying the chemical composition also affects the Weber number - the ratio of the momentum, or velocity, inertia to the surface tension - and in turn, affects the resulting droplet size distribution. Thus, it is possible that the results obtained in this work include both effects simultaneously. Also, an independent method of obtaining the droplet size distribution was not conducted. Throughout this paper the word diffusivity means "apparent" diffusivity. With this in mind, the results and their interpretations follow.

An emulsion of NGS solvent in V5/10 salt simulant was placed in a NMR tube. The tube was then placed in a 7 Tesla magnet. The emulsion was irradiated with radio waves and the residual magnetization was recorded after a short wait. The "short" wait must not be larger than half the square of the mean droplet radius divided by the diffusivity. Processing of the data yielded the average diffusivity, " $D$ ", of the solvent components (mostly Isopar ${ }^{\circledR} \mathrm{L}$ ) suspended in the salt simulant. The solvent diffusivity (assumed to be constant) was measured for the eight solvents against the salt simulant and the boric acid and they are listed in Table 3-14. Table 3-14 also lists the recorded temperature of the sample during the measurements. As can be observed, the highest temperature difference between maximum and minimum temperature was less than one degree. Thus, no attempts were made to apply a temperature correction to the data. The data of Table 3-14 was used to analyze whether the measured diffusivity correlated with compositional variances. A first approach or qualitative way of determining which chemical components affect the diffusivity data is using polynomial fitting in conjunction with the design matrix discussed earlier. Figure 3-3 and Table 3-15 show the results of a polynomial fitting and the results indicate that three out of four components in the solvent such as TiDG, Isopar ${ }^{\circledR} \mathrm{L}$, and the extractant affected the diffusivity. The most impacting parameter is the TiDG. 
Table 3-14. NGS diffusivity $\left(\mathrm{m}^{2} / \mathrm{s}\right)$ in Salt Simulant and Boric acid diffusivities

\begin{tabular}{|c|c|c|c|c|}
\hline \multirow{2}{*}{ Solvent } & \multicolumn{2}{|c|}{ Diffusivity $\left(\mathrm{m}^{2} / \mathrm{s}\right)$} & $\begin{array}{c}\text { V5/10 Salt } \\
\text { Simulant } \\
\text { Temperature } \\
\left({ }^{\circ} \mathrm{C}\right)\end{array}$ & $\begin{array}{c}\text { Boric Acid } \\
\text { Temperature } \\
\left({ }^{\circ} \mathrm{C}\right)\end{array}$ \\
\hline NGS-1 & $1.14 \mathrm{E}-10$ & Boric Acid & 21.0 & 21.8 \\
\hline NGS-2 & $1.23 \mathrm{E}-10$ & $1.58 \mathrm{E}-9$ & 21.2 & 21.7 \\
\hline NGS-3 & $1.02 \mathrm{E}-10$ & $1.21 \mathrm{E}-9$ & 21.6 & 21.6 \\
\hline NGS-5 & $1.05 \mathrm{E}-10$ & $3.09 \mathrm{E}-10$ & 21.2 & 21.6 \\
\hline NGS-6 & $1.14 \mathrm{E}-10$ & $2.35 \mathrm{E}-10$ & 21.4 & 21.7 \\
\hline NGS-7 & $5.96 \mathrm{E}-11$ & $1.37 \mathrm{E}-9$ & 21.4 & 21.3 \\
\hline NGS-11 & $2.91 \mathrm{E}-10$ & $9.8 \mathrm{E}-10$ & 21.3 & 21.3 \\
\hline NGS-13 & $1.61 \mathrm{E}-10$ & foamed & 21.6 & 21.1 \\
\hline NGS-18 & $2.62 \mathrm{E}-10$ & foamed & 21.6 & 21.0 \\
\hline
\end{tabular}

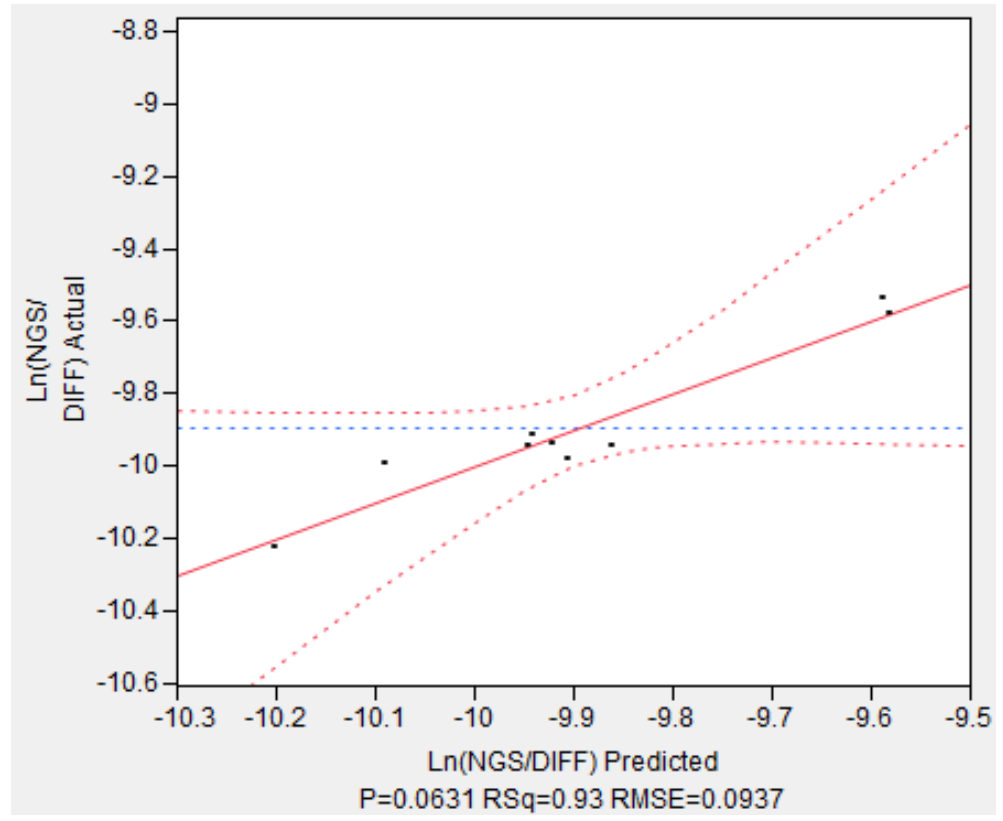

Figure 3-3. Fitting results of the $\log D_{\text {Isopar }}^{\circledR}{ }_{L}$ of NGS droplets in Salt Simulant

Table 3-15 Relevant parameters determined from the fit in Fig. 3.3

\begin{tabular}{|c|c|c|c|c|}
\hline Term & Estimate & Std Error & t Ratio & Prob $>|t|$ \\
\hline Intercept & -8.692385 & 0.346758 & -25.07 & $0.0001^{*}$ \\
\hline TiDG & 56.902342 & 14.8942 & 3.82 & $0.0316^{\star}$ \\
\hline MaxCalix & 7.8822758 & 2.018765 & 3.90 & $0.0298^{*}$ \\
\hline Isopar L & -5.839861 & 1.789032 & -3.26 & $0.0470^{*}$ \\
\hline$(\text { TiDG }-0.0025)^{\star}($ TiDG -0.0025$)$ & -62122.41 & 16053.1 & -3.87 & $0.0305^{\star}$ \\
\hline$(\text { MaxCalix }-0.025)^{*}($ Isopar L-0.18801) & -184.1048 & 70.06345 & -2.63 & 0.0785 \\
\hline
\end{tabular}




\subsection{Diffusivity Measurement: NGS Droplets in $10 \mathrm{mM}$ Boric Acid}

Diffusivity analysis of the NGS in boric acid emulsions is shown in Table 3-16. As can be seen in Table 3-16, all the NGS components affected the Isopar ${ }^{\circledR} \mathrm{L}$ diffusivity constant. The modifier, TiDG, and MaxCalix (extractant) negatively impacted the measured apparent diffusivity constant. This effect might be due to the favorable interaction between these polar molecules and water. However, from Table 3-16 having more Isopar ${ }^{\mathbb{B}} \mathrm{L}$ in the solvent increased the diffusivity.

Table 3-16. Relevant parameters determined from fitting $\log D_{\text {Isopar }^{\circledR} L}$ in

Boric Acid. $\mathbf{D}$ = Diffusivity

Term
\begin{tabular}{|l|r|r|r|r|}
\hline Intercept & -8.87819 & 0.021059 & -421.6 & $0.0015^{*}$ \\
mod & -0.432433 & 0.033775 & -12.80 & $0.0496^{\star}$ \\
TiDG & -19.30931 & 1.830062 & -10.55 & 0.0602 \\
MaxCalix & -14.36641 & 0.200556 & -71.63 & $0.0089^{*}$ \\
Isopar L & 3.0727762 & 0.079757 & 38.53 & $0.0165^{\star}$ \\
(mod-0.60714)*(TiDG-0.00214) & 991.64362 & 16.80721 & 59.00 & $0.0108^{*}$ \\
\hline
\end{tabular}

\subsection{Diffusivity Measurement: Salt Simulant or Boric Acid Droplets in NGS Solvent}

The measured diffusivity results for emulsions of salt simulant droplets in the solvents are shown in Table 3-17 and their corresponding polynomial fitting in Table 3-18. As can be seen from Table 3-17 both the modifier and Isopar ${ }^{\circledR} \mathrm{L}$ affected the measured apparent diffusivity in the salt simulant droplets.

Table 3-17 also shows the apparent diffusivity data from the emulsion of boric acid droplets in the solvents. The low diffusion constants observed with samples NGS-1, NGS-3 and NGS-7 is due to the absence of TiDG. When TiDG was present in the solvent, higher diffusivity constants were measured. This may be due to the higher Weber numbers expected when ionized TiDG weakens the interfacial tension between the solvent and boric acid. 
Table 3-17. Salt simulant and boric acid diffusivity $\left(\mathrm{m}^{2} / \mathrm{s}\right)$ in NGS solvent after dispersion

\begin{tabular}{|c|c|c|c|c|}
\hline Solvent & $\begin{array}{c}\text { Diffusivity of } \\
\text { V-5/10 Salt } \\
\text { simulant }\left(\mathrm{m}^{2} / \mathrm{s}\right)\end{array}$ & $\begin{array}{c}\text { Diffusivity of } \\
\text { Boric Acid* } \\
\left(\mathrm{m}^{2} / \mathrm{s}\right)\end{array}$ & $\begin{array}{c}\text { Boric Acid } \\
\text { Temperature } \\
\left({ }^{\circ} \mathrm{C}\right)\end{array}$ & $\begin{array}{c}\text { V5/10 Salt } \\
\text { Simulant } \\
\text { Temperature } \\
\left({ }^{\circ} \mathrm{C}\right)\end{array}$ \\
\hline NGS-1 & Foamed & $3.34 \mathrm{E}-10$ & 21.8 & 21 \\
\hline NGS-2 & $8.65 \mathrm{E}-11$ & $1.16 \mathrm{E}-9$ & 21.7 & 21.2 \\
\hline NGS-3 & $7.58 \mathrm{E}-11$ & $8.97 \mathrm{E}-10$ & 21.6 & 21.6 \\
\hline NGS-5 & $1.10 \mathrm{E}-10$ & $1.17 \mathrm{E}-9$ & 21.6 & 21.2 \\
\hline NGS-6 & $1.16 \mathrm{E}-10$ & $9.18 \mathrm{E}-10$ & 21.7 & 21.4 \\
\hline NGS-7 & $1.21 \mathrm{E}-10$ & $1.05 \mathrm{E}-9$ & 21.3 & 21.4 \\
\hline NGS-11 & $1.5 \mathrm{E}-10$ & $1.2 \mathrm{E}-9$ & 21.3 & 21.3 \\
\hline NGS-13 & $1.29 \mathrm{E}-10$ & $1.27 \mathrm{E}-9$ & 21.1 & 21.6 \\
\hline NGS-18 & $1.46 \mathrm{E} 10$ & Foamed & 21 & 21.6 \\
\hline
\end{tabular}

*Please note that dilute boric acid has a lower viscosity than salt simulant that contains a high concentration of hydroxides

Table 3-18. Relevant parameters determined from fitting $\log D_{\text {salt simulant }}$ in

NGS solvent.

Term
\begin{tabular}{|l|r|r|r|r|}
\hline Intercept & -21.15553 & 2.35917 & -8.97 & $0.0122^{*}$ \\
mod & 5.2461423 & 0.8241 & 6.37 & $0.0238^{*}$ \\
TiDG & 41.920199 & 21.07843 & 1.99 & 0.1850 \\
Isopar L & 36.93296 & 9.025811 & 4.09 & 0.0549 \\
$($ mod-0.64125)*(TiDG-0.00281) & 1550.6684 & 321.247 & 4.83 & $0.0403^{*}$ \\
(TiDG-0.00281)*(Isopar L-0.21152) & 12204.259 & 3605.222 & 3.39 & 0.0773 \\
\hline
\end{tabular}

Another organic liquid physical property that plays a role in particle-particle coalescence and mass transfer is viscosity. Viscosity is related to momentum transfer by translation of the molecules and their mutual collisions that in the case of liquids are very frequent. Viscosity of the continuous medium, in this case an aqueous solution, mitigates the speed of approach between droplets. However, once the distance between droplets is at or below a minimum critical distance, the kinetics of coalescing is affected by additional factors that include the rate of aqueous solution drainage from the interstitial space between the droplets and the deformation ability (bending and pinching off) of the droplet's surfaces to bridge with other droplets. How fast a droplet's surface deforms and relaxes relates to its interior viscosity. Just like diffusivity in constrained spaces differs from bulk diffusivity so does viscosity. Before discussing how viscosity varies with chemical variations, a more detail analysis of the diffusivity data of each component in the solvent is required.

The diffusivity analysis Isopar ${ }^{\circledR} \mathrm{L}$ diffusivity spans a wider range than any of the other NG-CSSX components since it is a mixture of hydrocarbons. These hydrocarbons can be 
identified by obtaining the H-NMR spectrum of the component at their measured diffusivity. The NMR experiment can resolve (separate) the components of a mixture by their diffusivity (or speed of movement in the mixture). Different molecular size and structure gives molecules different translational or rotational rate that can be used to obtain the identity of that molecule. As discussed for Fig. 2.2, the same analysis is applied to the diffusivity measurements of the NGS components. Shown in Fig. 3-4 are the H-NMR spectra of NGS and the corresponding diffusion rates of NGS components. Fig 3-4 to Fig 3-6 show the diffusivity (abcissa scale) as measured by HNMR versus chemistry (ordinate scale) correlation data for salt solution dispersed in NG-CSSX. The horizontal line shown in these figures indicates the measured diffusivity value (abscissa) and the corresponding H-NMR spectrum that has this diffusivity is shown in red color. This figures aids in connecting chemistry (the make-up of the molecule as presented by the spectrum) and the corresponding diffusivity (a value typical used in mass transfer engineering). As can be seen in Fig. 3-4, most of the Isopar ${ }^{\mathbb{B}} \mathrm{L}$ is made up of straight chain aliphatic possibly dodecane and lower straight chains $\left(\mathrm{CH}_{3}\right.$ and $\mathrm{CH}_{2}$ peaks) diffusing at $3.8 \mathrm{E} 10 \mathrm{~m}^{2} / \mathrm{s}$. But the diffusion histogram (shown in the abscissa) shows a broad shoulder towards the slower diffusion side of the scale. As shown in Fig. 3-5, the $\mathrm{CH}_{2}$ peak (at $0.5 \mathrm{ppm}$ when adjusted this peak should at $1.2 \mathrm{ppm}$ ) gets taller probably due to chain lengths longer than $\mathrm{C} 12$ (or $\mathrm{CH}_{3}-\mathrm{O}$ but this is less likely). The slowest of the Isopar ${ }^{\circledR} \mathrm{L}$ component is shown in Fig. 3-6 with a single peak near 0 ppm. The single peak is possibly due to cyclic molecules like cyclopropane (although it is lighter than C12, its volume slows this molecule translation rate down). Finally, Fig. 3-7 shows the rotational rate of the $\mathrm{OH}$ group in the modifier. This rotation is faster than any molecular group in the modifier.

These spectra can be quantified (via signal integration) to give an estimate of the composition of Isopar ${ }^{\circledR} \mathrm{L}$ as a function of hydrocarbon chemistry and structure. Integrating the peaks shown in Fig. 3-7 yields the relative amount of branched and saturated rings in Isopar ${ }^{\mathbb{B}} \mathrm{L}$. In the case of the modifier, this method can calculate the concentration of the modifier's isomer that contains an ethanol group. In addition to this, this method can provide the droplet size distribution (in this case for dispersed salt solution in NG-CSSX) without diluting the sample as typically is done with other routine droplet size distribution methods. A sample of this calculation is shown in Appendix A (Fig. A2) where an uniform Gaussian distribution was used to fit the data shown in Fig. A1. As can be seen in Fig. A2, a monodispersed particle size fit the residual magnetization at low magnetic field gradients. At higher magnetic field gradient, a monodispersed PSD did not fit the data well.

With the diffusivity data presented in Fig. 3-4 to Fig. 3-7, the viscosity of the interior of an NGS droplet can be estimated. More precisely, the diffusivity data in conjunction with the molecular size yield the solvent viscosity as shown in Eq. $13 .{ }^{27}$ Equation 13 is the Wilke-Chang equation estimate of the viscosity of a binary solvent. This equation applies when the dispersed component molal volume is less than $0.5 \mathrm{~m}^{3} / \mathrm{mol}$ 
$D \times \eta_{\text {solvent }}=\frac{1.173 \times 10^{-16} \times \text { Temperature } \times \text { Solvent Molecular Weight }{ }^{1 / 2}}{\text { Solvent molar Volume }}$

EQ. 13

In Eq. 13, the symbol " $D$ ", " $\eta "$, and " $T$ " stand for diffusivity, viscosity of the solvent, and temperature. The molar volume was obtained from using group contribution theor ${ }^{28}$

Figure 3-4. H-NMR spectrum of the component in Isopar ${ }^{\circledR} L$ that diffuses at $3.89 \mathrm{E}-10$ $\mathrm{m}^{2} / \mathrm{s}$

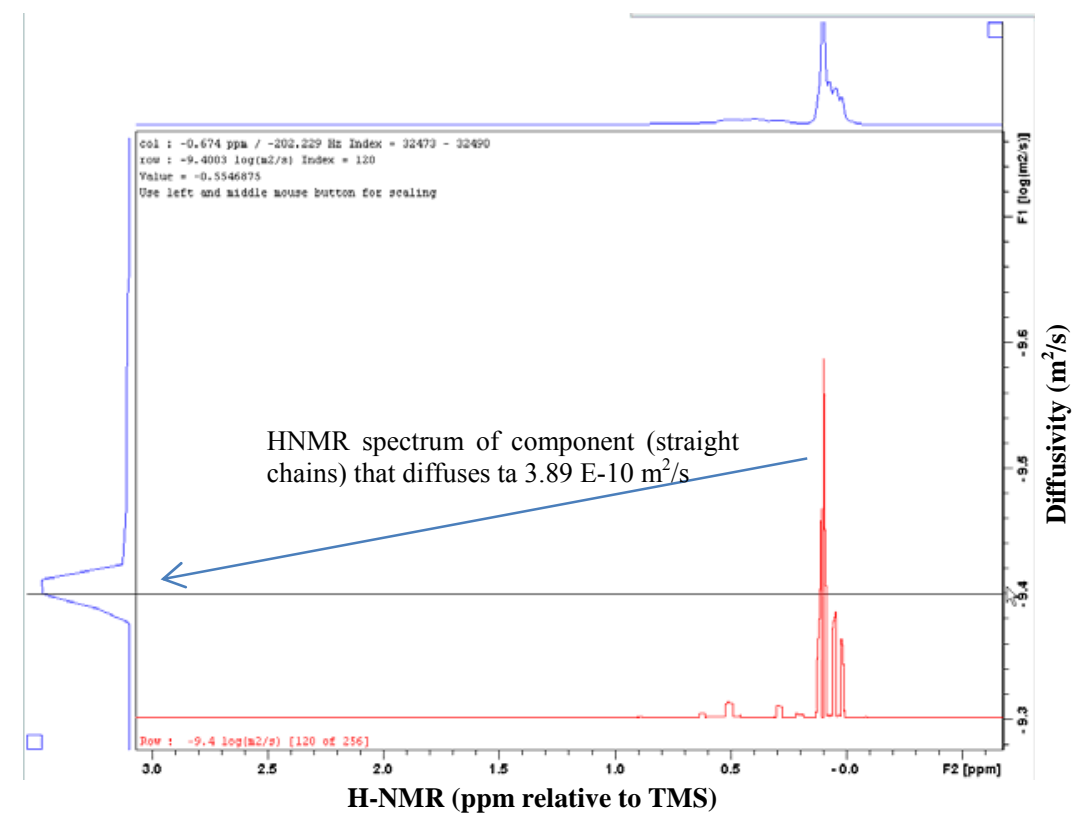

.Figure 3-5. H-NMR spectrum of the component in Isopar ${ }^{\circledR} L$ that diffuses at $3.47 \mathrm{E}-10$ $\mathbf{m}^{2} / \mathbf{s}$

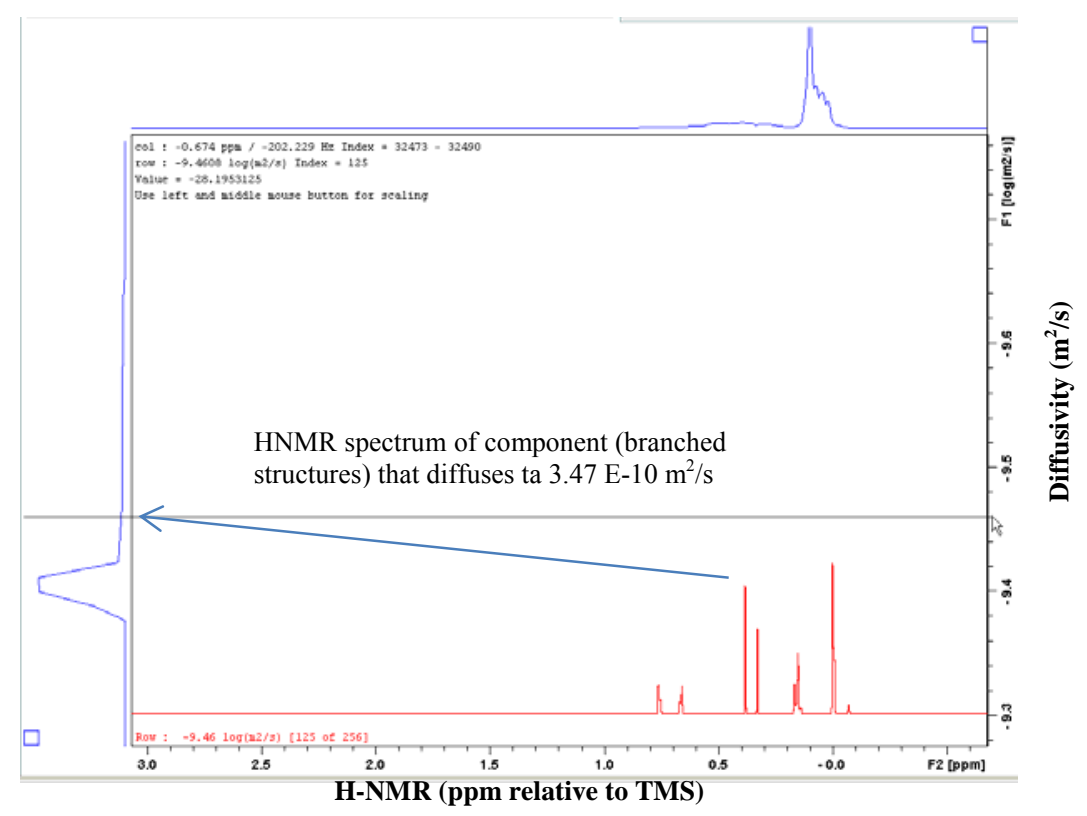


Figure 3-6. H-NMR spectrum of the component in Isopar $^{\circledR} L$ that diffuses at $2.29 \mathrm{E}-10 \mathrm{~m}^{2} / \mathrm{s}$

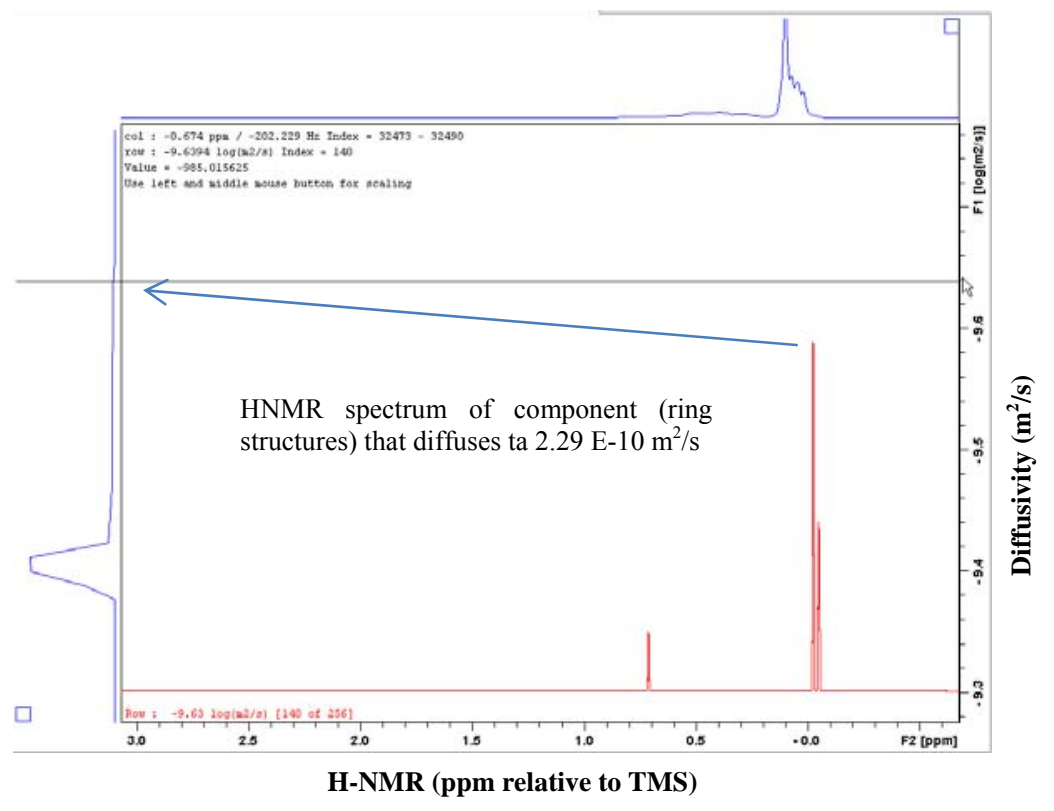

Figure 3-7. Histogram (or concentration) of diffusing species in salt solution/NGS emulsions

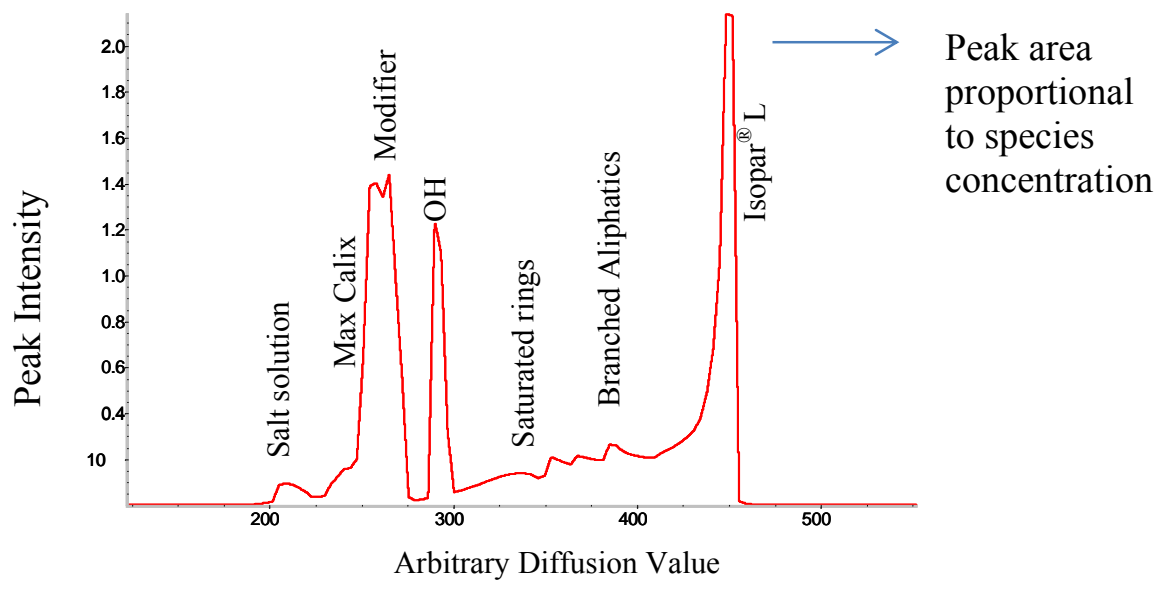

For components with molar volumes larger than $0.5 \mathrm{~m}^{3} / \mathrm{mol}$ the Wilke-Chang equation cannot predict their diffusivity (or viscosity) accurately. Under this condition, the Einstein-stoke equation has better accuracy (shown in EQ. 14).

$D \times \eta=\frac{K T}{6 \times \pi \times r}$

EQ. 14 
A calculation of the molar volume ${ }^{28}$ of the four components that make up NGS is shown in Table 3-19. As shown in Table 3-19, both the suppressor and the extractant can be considered as large particles dispersed in the Isopar ${ }^{\circledR} \mathrm{L} /$ modifier blend.

Table 3-19. Estimated molecular size of the NGS components

\begin{tabular}{||l|c|l||}
\hline Component & $\begin{array}{l}\text { Estimated Molar } \\
\left.\text { Volume } \mathbf{( m}^{\mathbf{3}} / \mathbf{m o l}\right)\end{array}$ & $\begin{array}{l}\text { Likely Applicable } \\
\text { Viscosity Equation* }\end{array}$ \\
\hline Isopar $^{\circledR}$ L & 0.185 & Wilke-Chang \\
\hline TiDG & 0.715 & Stokes-Einstein \\
\hline MaxCalix & 1.25 & Stokes-Einstein \\
\hline Modifier (Cs-7SB) & 0.266 & Wilke-Chang \\
\hline
\end{tabular}

*Molar volumes greater than 0.5 is considered large particles dispersed in monomeric solvent ${ }^{29}$

Based on the estimates shown in Table 3-19, the viscosity of each component and that of the Isopar ${ }^{\circledR} \mathrm{L} /$ modifier blend can be estimated with the appropriate transport equation (EQ. 13 or 14). To calculate viscosity with EQ. 13 or 14 we need an estimate of the diffusivity. Using the data shown in Fig. 3-4 through 3-6, the calculated viscosities are shown in Table 3-20.

Table 3-20. Estimated viscosity of each of the NGS components

\begin{tabular}{|c|c|c|c|c|}
\hline Quantity & Isopar $^{\circledR} \mathrm{L}^{*}$ & Modifier & MaxCalix & TiDG \\
\hline${\text { Unrestricted } D\left(\mathrm{~m}^{2} / \mathrm{s}\right)^{\&}}^{\text {Q }}$ & $3.8 \pm 0.3 \mathrm{E}-10$ & $9.3 \pm 1 \mathrm{E}-11$ & $7.5 \pm 4 \mathrm{E}-11$ & $2.5 \mathrm{E}-11$ \\
\hline$r(\mathrm{~m})^{\#}$ & $\sim 4.20 \mathrm{E}-10$ & $4.72 \mathrm{E}-10$ & $7.91 \mathrm{E}-10$ & $6.57 \mathrm{E}-10$ \\
\hline Viscosity (cP) from Eq. 13 & 0.147 & 0.41 & 0.4 & 0.67 \\
\hline Viscosity (cP) from Eq. 14 & 1.37 & 4.96 & 3.67 & 13.3 \\
\hline
\end{tabular}

${ }^{\#}$ Assuming spherical geometry. Isopar ${ }^{\circledR} \mathrm{L}$ is mostly a linear chain.

*Assuming straight $\mathrm{C} 12$ long chains are the most abundant. Isopar ${ }^{\circledR} \mathrm{L}$ viscosity measured $0.1278 \mathrm{cP}$ (Exxon-Mobil MSDS 2008)

${ }^{\$}$ Calculation done at $22{ }^{\circ} \mathrm{C}$ from Eq. 13 and Eq. 14

${ }^{\&}$ Obtained from the ordinate peaks in Figures 3-4, 3-5, 3-6, and 3-7.

A glance at Table 3-20 indicates the viscosity calculated from the Isopar ${ }^{\circledR}$ L and TiDG is less than that calculated from the modifier and the extractant. The assumption of using dodecane for Isopar ${ }^{\circledR} \mathrm{L}$ may be affecting the calculation accuracy. Similarly, the molecular volume calculation assumes the molecules to be spherical. Thus, a molecule like TiDG which has a star shape is seen by the calculation to be a long linear molecule. The values listed in Table 3-20 bounds the measured values of $2.7 \mathrm{E}-3$ and $3.5 \mathrm{E}-$ $3 \mathrm{~kg} / \mathrm{m}^{*} \mathrm{~s}$ for a similar solvent composition at ORNL. If the diffusivity measurements were not available, the viscosity of a mixture (or emulsion in this case) can be estimated from the viscosity of each of the components that makes up the solvent using empirical equations available in the literature ${ }^{30,31,32,33,34,35,36 \text {, and } 37}$. The rheological properties of the NGS as well as the CSSX solvent are influenced by their highest concentration components such as the modifier and Isopar $^{\circledR} \mathrm{L}$. 
Since no viscosity measurement was done with the samples listed in Table 3-1, we used data from the CSSX solvent (that contains a much higher modifier concentration) to identify an empirical formula that can predict viscosity for NGS. Using the viscosity data obtained from a CSSX solvent system published earlier where the CSSX solvent was measured during evaporation, ${ }^{38}$ the intrinsic viscosity of the modifier (containing trace amounts of extractant and suppressor) was estimated from several empirical formulas that estimate the viscosity of mixture as shown in Table 3-21. The model or models that predict the modifier viscosity the best is assumed to predict the viscosity of the blend and therefore, it can predict the NGS viscosity. As can be seen from Table 3-21, the measured solvent viscosities are of similar magnitude to that predicted by the EinsteinStokes equation in Table 3-20 for NGS. Please note that $1000 \mathrm{cP}$ (units used in Table 320) equals one $\mathrm{Pa}^{*} \mathrm{~s}(\mathrm{~kg} / \mathrm{m} * \mathrm{~s})$. Also note that the NGS viscosity is lower than that reported in Table 3-21 since NGS contains more Isopar ${ }^{\circledR} \mathrm{L}$ ( $74 \mathrm{wt} \%$ versus $69.1 \mathrm{wt} \%$ in CSSX).

Table 3-21 shows the estimate of the modifier viscosity from three different empirical formulas. The empirical formula developed by Kendall ${ }^{32}$ and $\mathrm{Kitano}^{37}$ estimated more precise values for the modifier viscosity but their accuracy has yet to be established. The value predicted by the Kendall expression of $30 \mathrm{cP}$ is more representative of honey like fluids. The Kendall expression $\eta_{\text {mix }}{ }^{1 / 3}=x_{\text {iso }} \eta_{\text {iso }}{ }^{1 / 3}+x_{\text {mod }} \eta_{\bmod }{ }^{1 / 3}$ has yet to fit a wider data range for the NGS solvent system. With one exception (an expression by $\mathrm{Guth}^{39}$ ), other expressions not shown in Table 3-21 but listed in the reference section failed to fit the measured solvent viscosity in Table 3-21. Using the Kendall expression, the Isopar ${ }^{\mathbb{R}} \mathrm{L}$, and modifier viscosity (from Table 3-21), the calculated viscosity for $0.5 \mathrm{M}$ modifier is $2.3 \mathrm{cP}$.

Table 3-21. The solvent (CSSX) viscosity in Pa*s of samples from Ref. 38 (1000 cP = Pa*s). Also shown the predicted modifier-extractant-suppressor combo viscosity

\begin{tabular}{|c|c|c|c|c|c|}
\hline $\begin{array}{c}\text { Solvent } \\
\text { Viscosity }\end{array}$ & Arrhenius $^{30}$ & Bingham $^{31}$ & Kendall $^{32}$ & Lederer $^{35}$ & Kitano $^{37}$ \\
\hline 0.00351 & 0.185 & $1.00 \mathrm{E}+04$ & 0.058 & 0.045 & 0.351 \\
\hline 0.00354 & 0.079 & $2.56 \mathrm{E}+04$ & 0.038 & 0.026 & 0.417 \\
\hline 0.00362 & 0.114 & $2.41 \mathrm{E}+04$ & 0.046 & 0.033 & 0.393 \\
\hline 0.00384 & 0.087 & $2.69 \mathrm{E}+04$ & 0.041 & 0.029 & 0.433 \\
\hline 0.00407 & 0.079 & $2.89 \mathrm{E}+04$ & 0.039 & 0.027 & 0.459 \\
\hline 0.00439 & 0.068 & $3.16 \mathrm{E}+04$ & 0.037 & 0.026 & 0.496 \\
\hline 0.00468 & 0.069 & $3.30 \mathrm{E}+04$ & 0.038 & 0.027 & 0.513 \\
\hline 0.00521 & 0.060 & $3.67 \mathrm{E}+04$ & 0.036 & 0.025 & 0.559 \\
\hline 0.00620 & 0.052 & $4.22 \mathrm{E}+04$ & 0.034 & 0.024 & 0.625 \\
\hline 0.01180 & 0.068 & $5.37 \mathrm{E}+04$ & 0.045 & 0.036 & 0.601 \\
\hline & & & & & \\
\hline Average & 0.086 & 31276.303 & 0.041 & 0.030 & 0.485 \\
\hline \%RSD & 45 & 37 & 17 & 22 & 19 \\
\hline
\end{tabular}

Isopar $^{\circledR} \mathrm{L}$ viscosity measured $0.001278 \mathrm{~kg} / \mathrm{m}^{\star} \mathrm{S}$ 
The empirical expression originally proposed for emulsions of polymers ${ }^{39}$ fit the data in Table 3-20 well. As shown in Table 3-22, the Guth's expression $\left(\eta_{\text {solvent }}=1+2.5 \phi\right.$ $+14.1 \phi^{2}$, where $\phi$ is the volume fraction of the dispersed phase assumed spherical in shape and the Isopar ${ }^{\circledR} \mathrm{L}$ viscosity is assumed to be unity) treats the modifier $(\phi)$ as a welldispersed insoluble phase. This assumption is not consistent with the high solubility of the modifier in Isopar ${ }^{\circledR} \mathrm{L}$ and yet this expression, derived for emulsions, by coincidence fit the data from Table 3-22 much better than simply taking the mean of the data. This coincidence proves once again that fitting does not imply causality but the expression may be used to guesstimate the viscosity of NGS solvent based on their modifier concentration. Since NGS solvent has much lower modifier concentration $(0.5 \mathrm{M}$ versus $0.75 \mathrm{M}$ in CSSX), the Guth's expression may reduce to the Einstein's equation ( $\eta_{\text {solvent }}=1$ $+2.5 \phi)$ if the Guth's expression still apply in that concentration range.

Table 3-22. Estimation of the solvent viscosity with the modified Einstein equation proposed by Guth ${ }^{39}$. All viscosity data shown in $\mathrm{cP}\left(\mathrm{mPa}^{*} \mathrm{~s}\right)$

\begin{tabular}{|c|c|c|}
\hline $\begin{array}{l}\text { Solvent } \\
\text { (Ref. 38) }\end{array}$ & Guth & Residual \\
\hline 3.51 & 2.84 & 0.67 \\
\hline 3.54 & 3.45 & 0.09 \\
\hline 3.62 & 3.24 & 0.38 \\
\hline 3.84 & 3.65 & 0.19 \\
\hline 4.07 & 3.96 & 0.11 \\
\hline 4.39 & 4.41 & -0.02 \\
\hline 4.68 & 4.65 & 0.03 \\
\hline 5.21 & 5.31 & -0.10 \\
\hline 6.20 & 6.38 & -0.18 \\
\hline 11.80 & 8.88 & 2.92 \\
\hline
\end{tabular}

In the case of predicting emulsion viscosity, several expressions already listed above can be used for prediction. Another empirical expression that contains more mathematical complexity relative to the continuous phase viscosity and, therefore, more fitting flexibility is the expression shown in Eq. 15. In this equation, $\mu_{\text {emulsion }}$ stands for the viscosity of the mixture, $\mu_{c \text { or } d}$ stands for the continuous and dispersed components in the mixture.

$\mu_{\text {emulsion }}=\left(\frac{\mu_{c}}{1-\emptyset_{d}}\right) \times\left(1+\frac{1.5 \times \mu_{d} \times \emptyset_{d}}{\mu_{c}+\mu_{d}}\right)$

EQ. 15

In EQ. $15, \phi_{d}$ stands for the volume fraction of the dispersed phase (" $d$ "). Using $\mu_{\mathrm{c}}=1$ or 1.2 for water or salt simulant respectively, $\mu_{\mathrm{d}}=2$ to $3 \mathrm{cP}$ for the NGS solvent, and if the $\phi_{\mathrm{d}}$ varies from 5 to $10 \%$, the expected emulsion viscosity ranges from 1.4 to $1.7 \mathrm{cP}$ depending on the value of $\phi_{\mathrm{d}}$. These calculated viscosities are close to the average value 
of the salt simulant and NGS viscosities. The magnitude of these viscosity values is sufficiently low for "unhindered" creaming (the gathering or concentrating of several droplets) to occur. Emulsions with fast creaming rates leads to faster coalescing or larger droplets that are easily push by a centrifuge force. However, spending a short residence time inside a centrifuge force and the emulsion may undergo an incomplete creaming process with a significant concentration of small droplets.

\subsection{Dynamic Interfacial Tension}

During emulsification, droplets undergo expansion, rotation, and eventual breakage. In those cases, the interface expands and contracts dynamically. In our case, our solvent is a blend of components. Our data indicates the suppressor affects the interfacial tension. We believe that since TiDG is slightly soluble in boric acid, the interface is dynamically changing as the TiDG diffuses into the aqueous portion of the interface. Similarly, the modifier, present in larger mass, diffuses into the aqueous phase and significantly affects the NGS-aqueous interface. In all these cases, the static interfacial tension measurement presented earlier may not describe the state of tension at the interface at equilibrium. A more appropriate measurement is a dynamic interfacial tension experiment that captures the dynamics of a changing interface.

There are several methods available for measuring the dynamic interfacial tension of emulsions. ${ }^{40}$ Most of these methods rely on measuring a single droplet (aqueous or organic) inside another insoluble phase. Emulsions are a collection of many different size droplets (mini and micro emulsions) that interact via Oswald ripening or aggregate and coalesce with each other. These mechanisms are interplays of adhesion forces, interfacial curvature, and hydrodynamics of the continuous phase. Therefore, a better method that utilizes a full emulsion and measures the average dynamic interfacial tension is magnetic resonance or NMR.

Early attempts to using NMR for this measurement relied on the Young-Laplace equation shown in Eq.16.

$$
\Delta P=4 \times \gamma / d
$$

EQ. 16

In Eq. 16, $\Delta \mathrm{P}$ stands for the pressure difference inside droplet relative to the outside of the droplet, $\gamma$ stands for the interfacial tension, and " $d$ " stands for the diameter of the droplet. By measuring the apparent diffusivity constant, one can obtain the mean droplet size distribution (and therefore the diameter " $d$ ") and knowing how the apparent diffusivity varies with pressure, one can estimate the pressure difference between the inside and the outside of the droplet. Plugging the pressure difference and the mean diameter into Eq. 16 and you will obtain the interfacial tension. However the results from this method lead to highly noisy data since at least two sources of noise contribute to the computed value. 
Another approach is to determine the diffusivity constant of NGS in aqueous solution and the diffusivity constant of a salt simulant or of boric acid in NGS. Using these two quantities, one can modify the static interfacial tension into a dynamic surface tension as shown in Eq. 17.

$$
\gamma_{\text {dynamic }}=\left(D_{\mathrm{NGS} \text { in salt simulant }} / D_{\text {salt simulant in NGS }}\right)^{n} * \gamma_{\text {static }}
$$

In Eq. 17, " $D$ " stands for the diffusion coefficient and " $n$ " is an empirical exponent whose magnitude indicates the extent and direction of a component diffusing into or out of the interface. ${ }^{19}$ The value of " $n$ " has been determined to be near 0.2 for components diffusing out of the interface. The diffusivity ratio and its exponent accounts for mass transfer between the interface and the two nearly immiscible liquids.

Assuming the apparent diffusion coefficient measured here for inside the droplets to be approximately the same as the molecular diffusion when the molecule is in the continuous phase (outside the droplet), one can estimate the dynamic interfacial tension with Eq. 17. Using the data in Table 3-1 that contains the static interfacial tension and the data in Table 3-12 that contain the diffusion data, the dynamic interfacial tension can be calculated. The calculated dynamic interfacial tension is shown in Table 3-23.

Table 3-23. The Calculated Dynamic Interfacial Tension between Salt Simulant and NGS Solvents $(\mathrm{NM}=$ Not Measured). All units are given in dynes/cm

\begin{tabular}{|c|c|c|}
\hline Solvent & $\begin{array}{c}\text { Static IFT } \\
\text { (dyn/cm) }\end{array}$ & $\begin{array}{c}\text { Dynamic } \\
\text { IFT } \\
\text { (dyn/cm) }\end{array}$ \\
\hline NGS-1 & 16.90 & Foamed \\
\hline NGS-2 & 15.01 & 16.11 \\
\hline NGS-3 & NM & NM \\
\hline NGS-5 & 17.14 & 16.98 \\
\hline NGS-6 & 16.64 & 16.58 \\
\hline NGS-7 & 16.45 & 14.28 \\
\hline NGS-11 & 15.44 & 17.63 \\
\hline NGS-13 & 17.09 & 11.27 \\
\hline NGS-18 & NM & NM \\
\hline
\end{tabular}

As can be seen from Table 3-23, the dynamic tension slightly differs from the static tension. In some cases, the dynamic tension trends in opposite direction to the static tension. The validity of this calculation must be confirmed with established methods for dynamic tension measurements. There is no commercially available emulsion system that will dynamically change during an interfacial measurement. Samples with these characteristic must be generated soon before measurement. 
One major assumption is the equality of diffusivity inside the droplet to its value outside. One can check this validity by calculating the diffusivity of TiDG or other solvent components in boric acid or salt simulant using the molar volume, molecular weight, and activity coefficients of these molecules. ${ }^{41}$ This procedure is involved and it includes a large number of assumptions.

\subsection{Phase Carry-Over Measurements}

Table 3-24 shows the relative concentration of boric acid that transferred to NGS after emulsification. The highest concentration of boric acid was observed in samples NGS-2, NGS-6, and NGS-13. An evaluation of these samples revealed that the modifier may be responsible for the abstraction of boric acid into the solvent as shown in Table 3-24. Although one of the sample tested (NGS-7) that contains a high concentration of modifier did not retain much boric acid, the statistical analysis still indicates the modifier plays a statistical role in the energetics of the surface with salt simulant. Inspection of Table 324 shows that both the modifier and TiDG increase the water content in NGS. The extractant lowers the boric acid content in NGS. This $\mathrm{pH}$ effect may be due to the low hydrogen bonding capability of the extractant.

In the case of the salt simulant, the glycolate groups in the calixarene may be stabilizing the water of the salt solution in the solvent. No correlation was found between the components of NGS and the concentration of NGS in boric acid.

Also shown in Table 3-24 is the concentration of Isopar ${ }^{\circledR} \mathrm{L}$ that transferred to the salt simulant or to the boric acid after emulsification. As can be seen from Table 3-24, when compared to the boric acid emulsions, more Isopar ${ }^{\circledR} \mathrm{L}$ transferred to the salt simulant since a higher whipping power (or higher applied Weber number) was applied during emulsification with the salt simulant. In this analysis, the cross product terms between the modifier and the extractant fit the data best.

Table 3-24. Percent Isopar ${ }^{\circledR} L$ in boric acid and in salt simulant after mixing

\begin{tabular}{|c|c|c|c|c|}
\hline sample & $\begin{array}{c}\text { \% Boric Acid in } \\
\text { NGS }\end{array}$ & $\begin{array}{c}\text { \% SS in } \\
\text { NGS }\end{array}$ & $\begin{array}{c}\text { \% Isopar }^{\circledR} \text { L in Boric } \\
\text { Acid }\end{array}$ & $\begin{array}{c}\text { \% Isopar } \\
\text { Simulant }\end{array}$ \\
\hline NGS-1 & 1.5 & 1.82 & 0.03 & 0.40 \\
\hline NGS-2 & 3.5 & - & 0.09 & 0.24 \\
\hline NGS-3 & 1.1 & 1.88 & 0.04 & 0.51 \\
\hline NGS-5 & 1.9 & 1.76 & 0.42 & 0.41 \\
\hline NGS-6 & 4.9 & 1.67 & 0.08 & 0.38 \\
\hline NGS-7 & 1.4 & 1.72 & 0.04 & 0.36 \\
\hline NGS-11 & 17 & 1.71 & Foamed & 0.36 \\
\hline NGS-13 & 5.1 & 1.85 & Foamed & 0.47 \\
\hline NGS-18 & Foamed & 1.23 & 0.18 & 0.29 \\
\hline
\end{tabular}


Statistical analysis of some of the data in Table 3-24 is shown in Table 3-25 and 3-26. According to Table 3-25, all three solvent components (TiDG, MaxCalix, and modifier) work in tandem to abstract boric acid into the NGS. Analysis of the output in Table 3-26 seems to indicate that MaxCalix appears to assist in the NGS carry over to the salt simulant. This may be provide evidence of the claims made in the recent Parson tests relative to the excess carry over they observed with the NGS-CSSX system.

Table 3-25. Boric acid concentration in NGS

Term
Intercept
Mod
Max
TiDG
(Mod- 0.64125$)^{\star}($ Max- 0.02188$)$
$(\text { Mod }-0.64125)^{\star}($ TiDG -0.00281$)$
$(\text { Max }-0.02188)^{\star}($ TiDG -0.00281$)$

$\begin{array}{rrrl}\text { Estimate } & \text { Std Error } & \text { t Ratio } & \text { Prob }>|\mathbf{t}| \\ -2.125824 & 0.687045 & -3.09 & 0.1990 \\ 13.396042 & 0.985565 & 13.59 & 0.0468^{*} \\ -87.80948 & 4.910037 & -17.88 & 0.0356^{*} \\ 447.79478 & 49.10037 & 9.12 & 0.0695 \\ -1046.341 & 43.76919 & -23.91 & 0.0266^{*} \\ 7983.4118 & 437.6919 & 18.24 & 0.0349^{*} \\ -49917.06 & 2188.459 & -22.81 & 0.0279^{*}\end{array}$

Table 3-26. The Effect of the NGS Components on NGS Solubility in Salt Simulant

Term
Intercept
Mod
Max
TiDG
(Mod-0.64125)*(Max-0.02188)
(Mod-0.64125)*(TiDG-0.00281)

$\begin{array}{rrrr}\text { Estimate } & \text { Std Error } & \text { t Ratio } & \text { Prob }>|\mathbf{t}| \\ 0.5487152 & 0.082758 & 6.63 & 0.0220^{*} \\ -0.229961 & 0.120968 & -1.90 & 0.1977 \\ 2.6161212 & 0.622076 & 4.21 & 0.0522 \\ -18.85121 & 6.220762 & -3.03 & 0.0938 \\ -21.29767 & 5.151943 & -4.13 & 0.0538 \\ 108.97667 & 51.51943 & 2.12 & 0.1687\end{array}$

The recent test conducted at Parsons ${ }^{2}$ where excess phase carry over was observed when the heavy phase flow was increased to the contactors. Thinking the problem was caused by the low MaxCalix and density, Parsons then added modifier to the NGS-CSSX solvent to approximate the original CSSX composition and operated the system at flow rates higher than $13 \mathrm{gpm}$ aqueous and $3 \mathrm{gpm}$ NGS-CSSX. They still observed what appeared to be foam leaving the heavy phase outlet of the weir. Yet, despite using a slightly different composition and operating conditions including a different size contactor, SRNL conducted hydraulic tests ${ }^{5}$ with a single stage centrifuge at comparable flow rates as the Parsons experiment and no foaming issues was observed in those tests.

From a chemical point of view, the solubility parameter of BOBCalixC6 and MaxCalix (according to additive group theory) is 61.9 and $46.1 \mathrm{MPa}^{1 / 2}$ respectively. The molal volumes are 302 and $364 \mathrm{~cm}^{3} / \mathrm{mol}$ respectively. The predicted SFT of BOBCalixC6 is slightly lower than that of MaxCalix. Thus, adding the same concentration of each to two different portions of the same solvent should have the same IFT. But adding MaxCalix at the $50 \mathrm{mM}$ level (seven times more than BOBCalixC6 is added to CSSX) may be 
impacting the abstraction of NGS-CSSX to the salt simulant. This high level of MaxCalix may be assisting the formation of stable micelle-like structures that traps air, salt simulant, and NGS-CSSX. The testing at SRNL proved that with one centrifuge stage, any liquid-liquid structure formed may be reversible and - based on the Parsons testing - that it becomes irreversible when more than one centrifuge unit process the fluid.

Incrementally adding the heavy phase to a steady system may change the particle size distribution of the emulsion (smaller droplets of the dispersed phase are made) and the viscosity of the emulsion increases perhaps beyond the point of diminishing the separation efficiency of the rotor. Decreasing the residence time of the fluids in the centrifuges also leads to the formation of small droplets and that may change the coalescence mechanism from inertia-driven creaming to Ostwald ripening (a much slower process).

\subsection{Conclusions}

Since 2008, the Savannah River Site has processed more than four million gallons of radioactive supernatant. To continue compliance with downstream processes, MCU is switching to a Blend solvent containing elements of the old CSSX solvent as well as newer, improved components. To complete implementation at MCU, additional work was needed to develop methods that can measure physical properties relevant to the performance of the new solvent. This work measured two relevant physical properties: static interfacial tension and diffusivity. Both properties play a relevant role in mixing and separating immiscible liquids. The work evaluated the impact of a chemistry change on these physical properties to gage the sensitivity of the system to the loss of chemical components.

This work found that the suppressor, TiDG, when it contacts $10 \mathrm{mM}$ boric acid ionizes and lowers the interfacial tension between NGS-CSSX and boric acid. The interfacial tension lowering is not sufficient to stop NGS-CSSX droplet coalescing. It was also found that the modifier enhances solvent carry over in the salt simulant probably through the alcohol group in the modifier molecule. The extractant also assisted in the salt simulant carry-over of the NGS-CSSX after contacting salt simulant.

Several literature-published adsorption models were evaluated for fitting the measured static surface tension data. It was found that both empirical and thermodynamically derived models fit the data. Because of the limited dataset, a true discrimination of the fitting models was not possible.

It was also found that increasing the Isopar ${ }^{\circledR} \mathrm{L}$ concentration in NGS increased the selfdiffusivity of the NGS components in NGS-Salt simulant emulsions. The diffusion NMR method was able to show and determine the amount of branched and cyclic molecules in Isopar ${ }^{\mathbb{B}}$ L. The method provided an estimate of the NGS viscosity in NGS-salt solution emulsions. The method also gave an estimate of the dynamic surface tension of NGS droplets in NGS-salt solution emulsions. 


\subsection{Recommendations, Path Forward or Future Work}

- Completing the more expanded original sample matrix

- Measure the dynamic surface tension of the solvents studied here with traditional methods such at the bubble pressure method. This measurement will establish the validity of the method proposed here.

- Develop a method of determining the droplet size distribution from the NMR analysis without assuming a priori a distribution function.

- Develop a group contribution method to predict physical properties of the solvent from the molecular structure of the components. 


\subsection{References}

${ }^{1}$ R. A. Pierce, T. B. Peters, M. L. Crowder, T. D. Caldwell, D. J. Pak, S. D. Fink, R. W. Blessing, and A. L. Washington, "Demonstration of the Next-Generation Caustic-Side Solvent Extraction Solvent with 2-cm Centrifugal Contactors using Tank 49H Waste and Waste Simulant", SRNL-STI-2011-00589, November 2011.

${ }^{2}$ R. Lentsch, "Salt Waste Processing Facility - Next Generation Solvent Test Report," PRPT-J-00024, 31 January 2013, Parsons-SRS.

${ }^{3}$ Williams, N. J. and Moyer, B. A., " Temperature dependence of the Next Generation Caustic Side Solvent Extraction (NGS-CSSX) Process Solvent”, ORNL-LTRNGCSSX-012, August, 2011.

${ }^{4}$ Roach, B. D., Williams, N. J., Duncan, N. C., and Moyer, B. A., "Effect of Possible Impurities on the Performance of the Next Generation Caustic Side Extraction (NGCSSX) Process", ORNL-LTR-NGCSSX-013, August, 2011.

${ }^{5}$ Herman, D. T., Duignan, M. R., Peters, T. B., Poirier, M. R., and Fondeur, F. F., "Mass Transfer and Hydraulic Testing of the V-05 and V-10 Contactors with the Next Generation Solvent”, SRNL-STI-2013-00413, Rev. 0, July 2013.

${ }^{6}$ Fondeur, F. F. and Fink, S. D. "Task Technical and Quality Assurance Plan for Measuring the Relaxation Rate of Next Generation Solvent (NGS) Droplets in Aqueous Solution and Aqueous Solution Droplets in NGS”, SRNL-RP-2013-00351, June 2013.

${ }^{7}$ Lange's Handbook of Chemistry 15th ed. pp 5.134

${ }^{8}$ Herman, D. T., Duignan, M. R., Williams, M. R., Peters, T. B., Poirier, M. R., and Fondeur, F. F.," Mass Transfer and Hydraulic tesing of the V-05 and V-10 Contactors with the Next Generation Solvent," SrnL-STI-2013-00413, Rev. 0, July 2013.

9 K. M. L,Taylor-Pashow, T. B. Peters, F. F. Fondeur, T. C. Shehee, and A. L. Washington, "Determination of the Impact of Glycolate on ARP and MCU Operations," SRNL-STI-2012-00218, Rev. 1, December 2012.

${ }^{10}$ C. A. Nash, M. A. Norato, D. D. Walker, R. A. Pierce, R. A. Eubanks, J. D. Clark, W. M. Smith, Jr., S. L. Crump, D. Z. Nelson, S. D. Fink, T. B. Peters, C. G. May, D. T. Herman, "Examination of Organic Carryover from 2-cm Contactors to Support the Modular CSSX Unit," WSRC-TR-2005-00182, Rev. 0, April 2005.

${ }^{11}$ Holz, M. and Weingartner, H. "Calibration in Accurate Spin-Echo Self-Diffusion Measurements Using 1H and Less-Common Nuclei", Journal of Magnetic Resonance, Vol. 92, (1991) 115-125. 
${ }^{12}$ Birdwell, J. F. and Lee, D. L. "Properties determinations and preliminary Engineering Evaluation of the Next Generation CSSX Processing," ORNL-LTR-NGCSSX-004, December, 2010.

${ }^{13}$ J. F. Birdwell, D. L. Lee, S. H. Buffey, and P. L. Szczygiel, "Next Generation CSSX Mass Transfer Perfomance in a Patially Pumping 5-cm Centrifugal contactor", ORNLLTR-NGCSSX-015, September 2011.

${ }^{14}$ Moyer, B. A., Bonnesen, P. V., Delmau, L. H., Sloop, F. V., Williams, N. J., and Roach, B. D. "Next-Generation Caustic-Side Solvent Extraction (NGS) Process Chemistry Status," Independent Technical Review, ORNL and Argonne National Laboratory, May 28-30, 2013

${ }^{15}$ Krevelen, D. W., "Properties of Polymers: Their Estimation and Correlation with Chemical Structure," $2^{\text {nd }}$ edition, Elsevier, Amsterdam, 1976.

${ }^{16}$ Barton, A. F. M., "Handbook of Polymer-Liquid Interaction Parameters and Solubility Parameters," CRC Press, Boca Raton, FL, 1990.

${ }^{17}$ Drelich, J. and Miller, J. D. "Spreading Kinetics for low viscosity n-alkanes on a water surface as recorded by the high-speed video system," Annales Universitatis Mariae Curie-Sklodowska, Lublin, Polonia, Vol. LIV/LV,7, 1999.

${ }^{18}$ Girifalco, L. A. and Good, R. J. J. Phys. Chem. (1957), 61, 904.

${ }^{19}$ Ayirala, S. C., and Rao N. Dandina, "A new mechanistics Parachor model to predict dynamic interfacial tension and miscibility in multicomponent hydrocarbon systems," J. of Colloid and Interface Science, (2006), 299, 321-331.

${ }^{20}$ Jufu, F. Buqiang, L, and Zihao, W, "Estimation of Fluid-Fluid Interfacial Tensions of Multicomponent Mixtures," Chemical Engineering Science, (1986), Vol. 41, No 10, pp. 2673-2679

${ }^{21} \mathrm{Xu}$, Y., Zhu, H., and Yang, L., "Estimation of Surface Tension of Ionic LiquidCosolvent Binary Mixtures by a Modified Hildebrand-Scott Equation," J. of Chemical \& Eng. Data, 2013, 2260-2265.

${ }^{22}$ Szyskowski, B. Z. Phys. Chem. 1908, 64, 385.

${ }^{23} \mathrm{P}$. Sonawane and A. Kumar, "A new equation for the correlation of surface tensioncomposition data of solvent-solvent and solvent-fused salt mixtures" Fluid Phase Equilibria, 157 (1999) 17-18.

${ }^{24}$ Hyungchul, K. and Burgess, D. J., "Prediction of Interfacial Tension between Oil Mixtures and Water," Journal of Colloid and Interface Science, (2001), 241, 509-513. 
${ }^{25}$ Baharamian, A. "Mutual solubility-interfacial tension relationship in aqueous binary and ternary hydrocarbon systems," Fluid Phase Equilibria, (2009), 285, 24-29.

${ }^{26}$ Fieber, W, Hanfer, V., and Normand, V, "Oil Droplet Size Determination in Complex Flavor Delivery Systems by Diffusion NMR Spectroscopy", Journal of Colloid and Interface Science, 356 (2011) 422-428.

${ }^{27}$ Wilke, C. R., and Chang, P., A. I. Ch. E. J., 1955, Vol. 1, 264-266.

${ }^{28}$ G. Le Bas, "The Molecular Volumes of Liquid Chemical Compounds," New York, David McKay Co, Inc. 1915.

${ }^{29}$ C. J. Geankoplis, "Transport Processes and Unit Operations," Third Edition, Prentice Hall Int., 1993.

${ }^{30}$ S. Arrhenius, Z. Physik. Chem., (1887), Vol . 1, 285-287.

${ }^{31}$ E. C. Bingham, Am. Chem. Journal, (1905), Vol.4, 481-484.

${ }^{32}$ J. Kendall and K. P. Monroe, J. Ame. Chem. Soc., (1917), Vol. 39, 1787-1791.

${ }^{33}$ M. H. Rahmes and W. L. Nelson, Anal. Chem., (1948), Vol. 20, \#10, 912-915.

${ }^{34}$ C. S. Cragoe, Proc. World Petroleum Congress (London), (1933), Vol. II, 529-535.

${ }^{35}$ E. L. Lederer, Proc. World Petroleum Congrss (London), (1933), Vol. II, 526-529.

${ }^{36}$ R. E. Maples, PetroleumRefinery Process Economics ( $2^{\text {nd }}$ edition), Pennwell Books, (2000). ISBN 0-471-400065-3.

${ }^{37}$ R. C.Reid, J. M. Prausnitz, and B. E. Poling, The Properties of Gases and Liquids, McGraw-Hill, New York, $4^{\text {th }}$ edition (1987).

${ }^{38}$ D. D. Lee, J. F. Birdwell, P. V. Bonnesen, and B. A. Tomkins, , "Density Changes in the Optimized CSSX Solvent System," ORNL-TM-2002-204, November 2002.

${ }^{39}$ E. Guth and R. Simha, "An Formula for the Viscosity of Polymeric Suspensions: Viscosity of Concentrated Suspensions," Kolloid Z. (1936), Vol. 74, \#3, pp 266-269.

${ }^{40}$ Nino-Amezquita, O, Enders, S, Jaeger, T. P., and Eggers, R, "Measurement and Prediction of Interfacial Tension of Binary Mixtures", Ind. Eng. Chem. Res., 2010, Vol. $49,592-601$.

${ }^{41}$ D'Agostino, C, Mantel, M. D., Gladden, L. F., and Moggridge, G. D., "Prediction of Binary Diffusion Coefficients in Non-ideal Mixtures from NMR Data: Hexanenitrobenzene Near Its Consolute Point”, Chem. Eng. Sci. 66 (2011), 3898-3906. 
SRNL-STI-2013-00733

Revision 0

Appendix A. A Typical Exponential Decay fit to the NMR Data to extract the Diffusivity Constant 
Figure A-1. A typical normalized residual magnetization as a function of the imposed magnetic field gradient

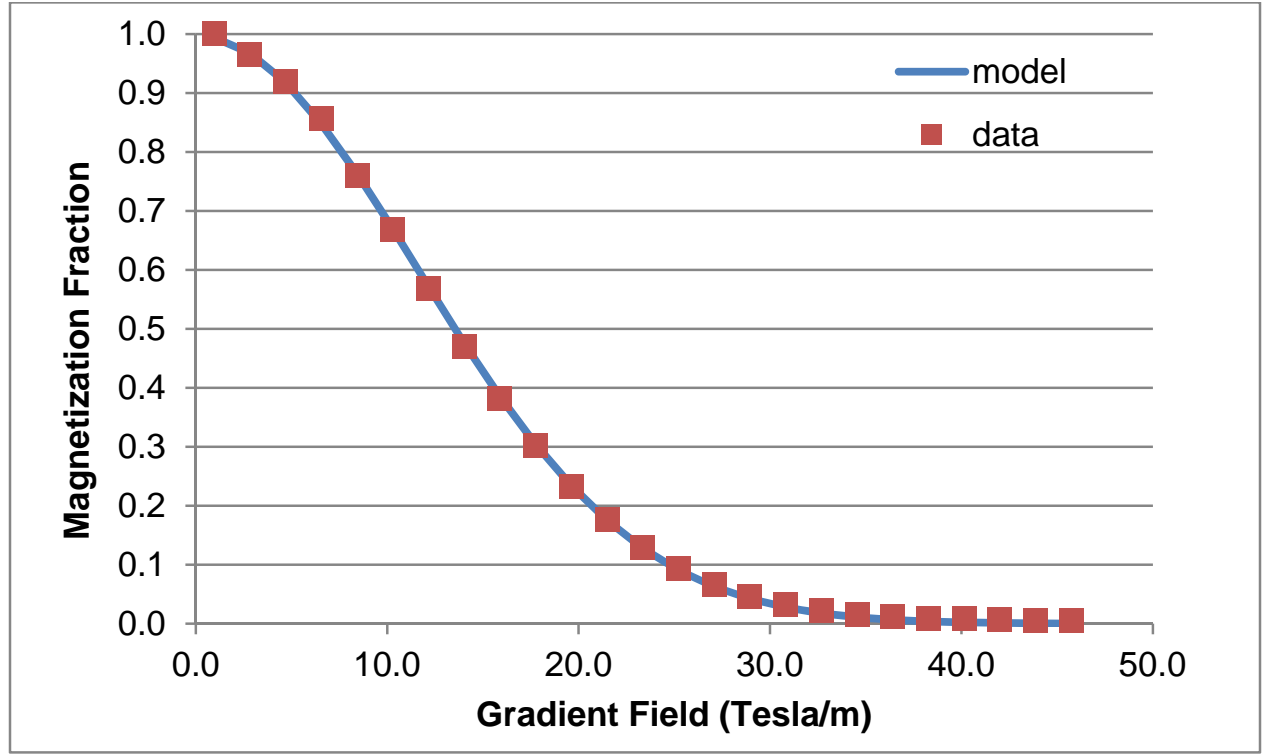

Figure A-2. Results of fitting a Gaussian particle size distribution with a mean of 1.4 microns and a dispersion of $\mathbf{0 . 2}$ microns

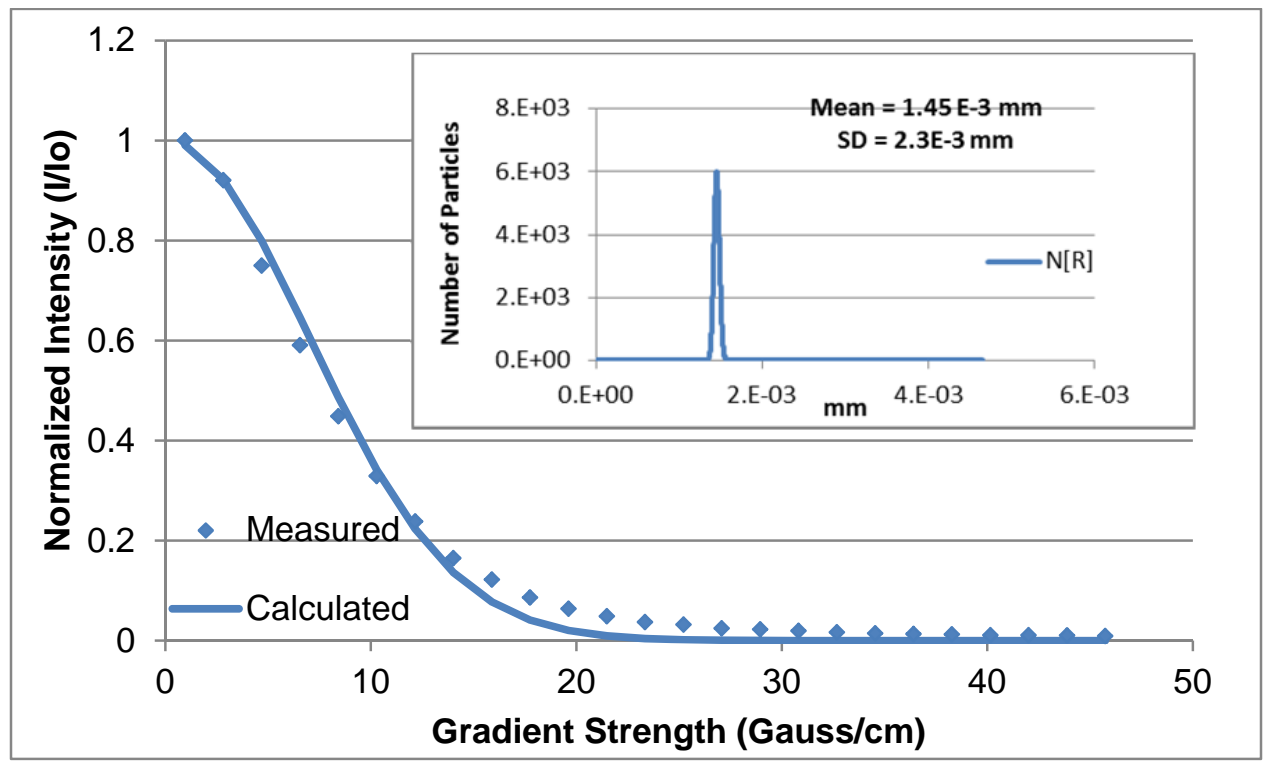




\section{Distribution:}

S. L. Marra, 773-A

T. B. Brown, 773-A

S. D. Fink, 773-A

F. M. Pennebaker, 773-42A

C. C. Herman, 773-A

E. N. Hoffman, 999-W

W. R. Wilmarth, 773-A

Records Administration (EDWS)

P. R. Jackson, DOE-SR, 703-46A 